\title{
OPEN A systematic review and meta-analysis of the serum lipid profile in prediction of diabetic neuropathy
}

\author{
Zixin Cai ${ }^{1,2}$, Yan Yang ${ }^{1,2}$ \& Jingjing Zhang ${ }^{1 凶}$
}

Whether the lipid profile in diabetic patients is associated with diabetic neuropathy (DN) development remains ambiguous, as does the predictive value of serum lipid levels in the risk of DN. Here, we performed the first meta-analysis designed to investigate the relationship between DN and the serum levels of triglyceride (TG), total cholesterol (TC), high-density lipoprotein cholesterol (HDL), and lowdensity lipoprotein cholesterol (LDL). Candidate studies were comprehensively identified by searching PubMed, Embase, Cochrane Library and Web of Science databases up to May 2020. Observational methodological meta-analysis was conducted to assess the relationships of TG, TC, HDL, and LDL levels with DN. Changes in blood lipids were used to estimate the effect size. The results were pooled using a random-effects or fixed-effects model. Potential sources of heterogeneity were explored by subgroup analysis. Various outcomes were included, and statistical analyses were performed using STATA (Version 12.0). Mean differences (MDs) and odds ratios (ORs) with $95 \%$ confidence intervals (CIs) were estimated. The Newcastle-Ottawa Scale (NOS) was applied to assess the methodological quality. 12 statistics were calculated to evaluate statistical heterogeneity. Funnel plots were utilized to test for publication bias. A sensitivity analysis was performed by omitting each study one by one. Thirty-nine clinical trials containing 32,668 patients were included in the meta-analysis. The results demonstrated that DN patients showed higher TG and lower $\mathrm{HDL}$ levels (MD $=0.34,95 \% \mathrm{Cl}: 0.20-0.48$ for TG; MD = $-0.05,95 \% \mathrm{Cl}:-0.08--0.02, I^{2}=81.3 \%$ for $\mathrm{HDL}$ ) than controls. Subgroup analysis showed that patients with type 1 diabetes mellitus (T1DM) neuropathy had elevated TG levels in their serum (MD $=0.25,95 \% \mathrm{Cl}: 0.16-0.35, I^{2}=64.4 \%$ for T1DM). However, only patients with T1DM neuropathy had reduced serum HDL levels, and there was no significant difference in serum HDL levels between patients with T2DM neuropathy and controls (MD $=-0.07,95 \% \mathrm{Cl}:-0.10-0.03, l^{2}=12.4 \%$ for T1DM; $\mathrm{MD}=-0.02,95 \% \mathrm{Cl}:-0.07-0.03, I^{2}=80.2 \%$ for T2DM). TC and LDL levels were not significantly different between $\mathrm{DN}$ patients and controls (MD $=-0.03,95 \% \mathrm{Cl}:-0.14-0.09, l^{2}=82.9 \%$ for TC; $\mathrm{MD}=-0.00,95 \%$ Cl: $-0.08-0.08, l^{2}=78.9 \%$ for $\left.L D L\right)$. In addition, compared with mild or painless $D N$ patients, those with moderate or severe pain DN pain had significantly reduced serum TC and LDL levels (MD $=-0.31$, 95\% Cl: $-0.49-0.13, I^{2}=0 \%$ for TC; MD $=-0.19,95 \% \mathrm{Cl}:-0.32--0.08, I^{2}=0 \%$ for LDL). TG levels and HDL levels did not vary considerably between patients with mild or painless $D N$ and those with moderate or severe $\mathrm{DN}$ pain patients $\left(\mathrm{MD}=0.12,95 \% \mathrm{Cl}:-0.28-0.51, I^{2}=83.2 \%\right.$ for TG; $\mathrm{MD}=-0.07,95 \% \mathrm{Cl}:-0.14-$ $0.01,1^{2}=58.8 \%$ for HDL). Furthermore, people with higher TG and LDL levels had higher risk of DN $\left(\mathrm{OR}=1.36,95 \% \mathrm{Cl}: 1.20-1.54, I^{2}=86.1 \%\right.$ for TG and $\mathrm{OR}=1.10,95 \% \mathrm{Cl}: 1.02-1.19, I^{2}=17.8 \%$ for LDL). Conversely, high serum HDL levels reduced the risk of DN $\left(\mathrm{OR}=0.85,95 \% \mathrm{Cl}: 0.75-0.96, I^{2}=72.6 \%\right)$, while TC levels made no significant difference with the risk of $D N(O R=1.02,95 \% \mathrm{Cl}: 1.00-1.04$, $I^{2}=84.7 \%$ ). This meta-analysis indicated that serum lipid profile changes are among the biological characteristics of DN. Lipid levels should be explored as routine laboratory markers for predicting the risk of DN, as they will help clinicians choose appropriate therapies, and thus optimize the use of available resources.

\footnotetext{
${ }^{1}$ National Clinical Research Center for Metabolic Diseases, Metabolic Syndrome Research Center, Key Laboratory of Diabetes Immunology, Ministry of Education, and Department of Metabolism and Endocrinology, The Second Xiangya Hospital of Central South University, Hunan 410011, China. ${ }^{2}$ These authors contributed equally: Zixin Cai andYanYang ${ }^{\varpi}$ email: Doctorzhangjj@csu.edu.cn
} 
Diabetic neuropathy (DN) is a highly common but often neglected complication of both type 1 diabetes mellitus (T1DM) and type 2 diabetes mellitus (T2DM), affecting an estimated $50 \%$ of individuals with diabetes ${ }^{1}$. Its prevalence is more than $2 \%$ in the general population ${ }^{2,3}$ and approximately $15 \%$ among people over 40 years old $^{4}$. Importantly, patients with peripheral and autonomic neuropathy have a more than twofold increase in their risk of death ${ }^{5}$. DN is a progressive and debilitating disease that can seriously reduce a patient's quality of life and is a key cause of non-trauma related amputations of the lower limbs ${ }^{6,7}$, which means that DN is recognized as the leading contributor to disability in people with diabetes ${ }^{8}$. In addition, one-third of subjects with DN report burning, tingling, shooting or lancing sensations as a symptom and need help alleviating this symptom ${ }^{9,10}$. Painful diabetic neuropathy (PDN) can seriously negatively affect patients' psychological and physical health, leading to anxiety, depression and sleep disorders ${ }^{11,12}$. The treatment of PDN can be a major challenge for both the clinician and the patient, as such pain is unresponsive or only partially responsive to existing management approaches ${ }^{13,14}$. Owing to the pressing need for a solution to DN, many clinical studies have been carried out to prevent or cure this complication. To date, there is no treatment to prevent the onset of DN other than intensive glycaemic control, which substantially reduces the incidence of DN only in T1DM patients and is minimally effective in preventing DN among patients with $\mathrm{T}_{2} \mathrm{DM}^{15-17}$. The cause of $\mathrm{DN}$ is more complex than dysregulated glucose levels alone. Even patients with good glycaemic control $(\mathrm{HbAlc}<5.4 \%)$ can still develop DN, suggesting that components other than glycaemic control may be involved in the onset and progression of $\mathrm{DN}^{18}$. Recently, some studies have implicated cardiovascular risk factors, such as obesity ${ }^{19}$ and triglycerides (TG $)^{20}$, in the pathogenesis of DN. Therefore, it is essential to understand whether lipid levels modulate DN progression.

Changes in serum lipid profiles and lipid metabolism are at the root of at least some disease mechanisms ${ }^{21}$. Numerous studies have confirmed that some biomarkers may be associated with DN, among which serum lipid levels might play a significant role. Despite the mass of evidence accumulated in the last few years and the considerable contribution of serum lipid profiles to $\mathrm{DN}$, there are still some considerable contradictions regarding the relationship between serum lipid levels and DN in observational and epidemiological studies ${ }^{23-26}$. Some studies have shown a positive association between a high level of total cholesterol (TC) and DN in diabetic patients ${ }^{27}$. In contrast, other studies have found a lack of significant changes in serum lipid profiles or even an inverse association between TG levels and $\mathrm{DN}^{18,28,29}$. Ascertaining whether a relation exists between serum lipid profiles and $\mathrm{DN}$ might lead to new disease-modifying therapies.

To comprehensively investigate the relationship between DN and the serum levels of TG, TC, high-density lipoprotein cholesterol (HDL), and low-density lipoprotein cholesterol (LDL), we conducted a meta-analysis quantitatively assessing the role of serum lipid levels in DN. The results provide new knowledge regarding the treatment of DN and may help in the development of clinical biomarker guidelines for DN.

\section{Methods}

Literature search strategy. This meta-analysis was conducted using the PRISMA (Preferred Reporting Items for Systematic Reviews and Meta-Analyses) guidelines ${ }^{30}$. This systematic review was prospectively registered in PROSPERO (CRD42020191400); the registration is available at http://www.crd.york.ac.uk/PROSPERO/ display_record.php?ID=CRD42020191400.

Relevant articles were identified through an electronic search of PubMed, Embase, Cochrane Library and Web of Science using the following search terms: ("Diabetic Neuropathy" OR "Neuropathies, Diabetic" OR "Neuropathy, Diabetic" OR "Diabetic Autonomic Neuropathy" OR "Autonomic Neuropathies, Diabetic" OR "Autonomic Neuropathies, Diabetic" OR "Diabetic Autonomic Neuropathies" OR "Neuropathies, Diabetic Autonomic" OR "Neuropathy, Diabetic Autonomic" OR "Diabetic Neuralgia” OR "Diabetic Neuralgias" OR "Neuralgias, Diabetic" OR "Diabetic Neuropathy, Painful” OR "Diabetic Neuropathies, Painful” OR "Neuropathies, Painful Diabetic" OR "Neuropathy, Painful Diabetic" OR "Painful Diabetic Neuropathies" OR "Painful Diabetic Neuropathy" OR "Neuralgia, Diabetic" OR "Symmetric Diabetic Proximal Motor Neuropathy" OR "Asymmetric Diabetic Proximal Motor Neuropathy" OR "Diabetic Asymmetric Polyneuropathy" OR "Asymmetric Polyneuropathies, Diabetic" OR "Asymmetric Polyneuropathy, Diabetic" OR "Diabetic Asymmetric Polyneuropathies" OR "Polyneuropathies, Diabetic Asymmetric" OR "Polyneuropathy, Diabetic Asymmetric" OR "Diabetic Mononeuropathy" OR "Diabetic Mononeuropathies" OR "Mononeuropathies, Diabetic" OR "Mononeuropathy, Diabetic" OR "Diabetic Mononeuropathy Simplex" OR "Diabetic Mononeuropathy Simplices" OR "Mononeuropathy Simplex, Diabetic" OR "Mononeuropathy Simplices, Diabetic" OR "Simplex, Diabetic Mononeuropathy" OR "Simplices, Diabetic Mononeuropathy" OR "Diabetic Amyotrophy" OR "Amyotrophies, Diabetic" OR "Amyotrophy, Diabetic" OR "Diabetic Amyotrophies" OR "Diabetic Polyneuropathy" OR "Diabetic Polyneuropathies" OR "Polyneuropathies, Diabetic" OR "Polyneuropathy, Diabetic") and ("serum lipid profiles" OR "lipid profiles" OR "lipid levels" OR "triglycerides" OR "total cholesterol" OR "high-density lipoprotein cholesterol" OR "low-density lipoprotein cholesterol"). Relevant articles published up to May 2020 were included in this study. We also manually screened the reference lists of retrieved articles to identify any potentially relevant studies. The summarized search strategy and the full electronic search strategies for multiple international databases are presented in Supplementary Files 2 and 3.

Selection criteria. Studies were included in this meta-analysis only if any all of the following criteria were met: (1) The study was published as an original article; (2) There were at least 2 groups (a DN group and a healthy control group); (3) The study evaluated the serum levels of TG, TC, HDL, or/and LDL of these 2 groups. Studies were excluded from this meta-analysis if any of the following criteria were met: (1) The study was a review, commentary, case report, case series or letter to the editor; (2) The study was performed in animals or in vitro; (3) The article was not in English (this restriction was imposed because English is the international language of science); (4) There was a significant difference in baseline age, gender, or body mass index (BMI) between the 


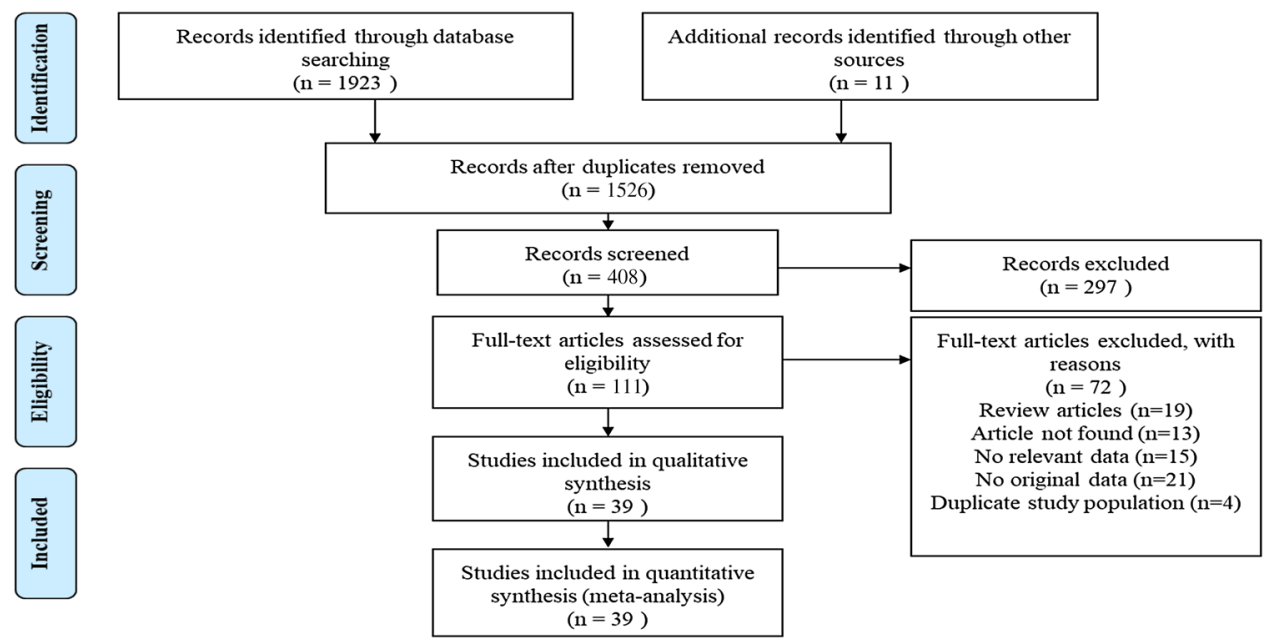

Figure 1. Flow of study selection.

2 groups. Studies were selected by two reviewers (ZC and YY) for inclusion in our analysis using the aforementioned criteria, and disagreements were resolved by consensus or with the help of a third reviewer (JZ).

Data extraction and quality assessment. Clinical information was robustly extracted from all eligible studies: number, first author, year, country, N (case/control), age, M\%, outcome reported, diabetes and Newcastle-Ottawa scale (NOS) score. Two investigators (ZC and YY) independently extracted study characteristics from the selected studies based on the predetermined inclusion and exclusion criteria. Any disagreements were settled with the help of a third reviewer (JZ) when necessary. For each study, the risk of bias was assessed using the NOS quality assessment instrument, which is used for assessing the quality of nonrandomized studies in a meta-analysis ${ }^{31}$. The measures on this scale comprise three items: the selection of participants, the comparability of cases and controls, and the ascertainment of outcomes. The scale has a minimum score of 0 and a maximum score of 9. Studies scoring at least 7 (corresponding to $78 \%$ of the maximum score) were regarded as having a low risk of bias ('good' quality); those that scored 4-6 were deemed to have a modest risk of bias ('fair' quality); and those that scored $<3$ were considered to have a substantial risk of bias ('poor' quality) ${ }^{32}$.

Statistical analysis. Statistical analysis was performed using the STATA software package (version 12.0, STATA Corp, College Station, TX). The results are expressed as mean differences (MDs) and odds ratios (ORs) with $95 \%$ confidence intervals (CIs). Lipid levels were extracted as continuous variables for statistical analysis and reported as the mean and standard deviation (SD). We also used the following approximations: if a study provided lipid levels with the mean and standard error, we converted the standard error into an SD by the following equation: standard error $\times$ square root of the sample size. If a study provided medians and interquartile ranges, we converted them to means and SDs as described by Hozo et al. ${ }^{33}$. For discrete data, if the OR and 95\% CI were not available, a $2 \times 2$ table was used to obtain the value of OR and $95 \%$ CI. A random-effects model was used to calculate the pooled results if the inconsistency index (I2) statistic was $>50 \%$, and a fixed-effects model was applied if $I 2 \leq 50 \%$. Data with $\mathrm{p} \geq 0.10$ and $I 2 \leq 50 \%$ were defined as having low heterogeneity. We assessed potential publication bias using funnel plots. Sensitivity analysis was performed by the leave-one-out method to assess whether the results were sufficiently robust and verify that they were not excessively influenced by any single study ${ }^{34}$.

\section{Results}

Study characteristics. We identified 1923 studies through electronic searches. We also identified and read 11 potentially relevant articles that we found by browsing the reference lists of related articles and reviews. Of the candidate studies, we excluded 1526 after reading the abstracts and titles because they were duplicate studies, review articles, animal studies, commentaries, proceedings, case observations, or irrelevant to the present analysis. By further analysing the full text of the 111 remaining papers, the remaining 39 eligible studies were included in our meta-analysis ${ }^{18,22-29,35-65}$. A flow chart showing our selection process is presented in Fig. 1 . Of these articles, 35 studies with 32,198 patients presented data for TG, 29 studies with 22,141 patients reported data for TC, 34 studies with 28,681 patients reported data for HDL and 30 studies with 22,615 patients presented data for LDL. There were 16 studies of patients with T2DM, 10 studies of patients with T1DM, 4 studies of patients with either T1DM or T2DM and 9 studies that did not specify the type of diabetes. Detailed characteristics of these eligible studies are described in Table 1.

Quality assessment. All thirty-nine studies had NOS quality scores greater than or equal to 5, indicating that all these studies had 'good' or 'fair' methodological quality. Details on the risk of bias among those 39 studies are summarized in Table 2 . 


\begin{tabular}{|c|c|c|c|c|c|c|c|c|}
\hline Number & References & Country & $\begin{array}{l}\mathrm{N} \text { (case/ } \\
\text { control) }\end{array}$ & Age & M\% & Study design & $\begin{array}{l}\text { Outcome } \\
\text { reported }\end{array}$ & Diabetes \\
\hline 1 & Jende $^{35}$ & Germany & $100(64 / 36)$ & $64.6 \pm 0.9$ & $68 \%$ & $\begin{array}{l}\text { cross-sectional } \\
\text { cohort }\end{array}$ & $\begin{array}{l}\text { TC, LDL-C, HDL- } \\
\text { C, TG }\end{array}$ & $\mathrm{T} 2 \mathrm{DM}$ \\
\hline 2 & Hosny $^{36}$ & Egypt & $60(30 / 30)$ & $51.238 \pm 7.784$ & NA & \begin{tabular}{|l|} 
a case control \\
study
\end{tabular} & $\begin{array}{l}\text { TC, LDL, HDL, } \\
\text { TG }\end{array}$ & T2DM \\
\hline 3 & Song ${ }^{29}$ & China & 455 & $62.8 \pm 8.61$ & $46 \%$ & $\begin{array}{l}\text { a case control } \\
\text { study }\end{array}$ & TC,TG,HDL,LDL & $\mathrm{DM}$ \\
\hline 4 & Vural $^{37}$ & Turkey & $165(90 / 75)$ & $64.0 \pm 10.3 / 66.6 \pm 14.7$ & $58.90 \%$ & $\begin{array}{l}\text { retrospective } \\
\text { study }\end{array}$ & $\begin{array}{l}\text { TC,TG,LDL- } \\
\text { C,HDL-C }\end{array}$ & $\mathrm{DM}$ \\
\hline 5 & Andersen $^{38}$ & Denmark & $144(27 / 117)$ & $62.59 \pm 17.31 / 52.36 \pm 17.52$ & $81.50 \%$ & $\begin{array}{l}\text { cross-sectional } \\
\text { study }\end{array}$ & $\begin{array}{l}\text { TC, LDL-C, HDL- } \\
\text { C, TG }\end{array}$ & T2DM \\
\hline 6 & $\begin{array}{l}\text { Mizokami- } \\
\text { Stout }^{39}\end{array}$ & USA & $5936(630 / 5306)$ & $39 \pm 18$ & $45 \%$ & \begin{tabular}{|l|}
$\begin{array}{l}\text { cross-sectional } \\
\text { study }\end{array}$ \\
\end{tabular} & $\begin{array}{l}\text { TG, HDL-C, LDL- } \\
\text { C,TC }\end{array}$ & T1DM \\
\hline 7 & Litzelman $^{40}$ & $\begin{array}{l}\text { African-Ameri- } \\
\text { can } 76 \%\end{array}$ & 352 & $60.4 \pm 9.6$ & $19 \%$ & $\begin{array}{l}\text { randomized } \\
\text { controlled trial }\end{array}$ & $\begin{array}{l}\text { HDL/cholesterol, } \\
\text { HDL,TG,TC }\end{array}$ & T2DM \\
\hline 8 & Aryan $^{41}$ & Iran & $939(444 / 495)$ & $43.28 \pm 14.42$ & $48 \%$ & $\begin{array}{l}\text { A nested case- } \\
\text { control stud }\end{array}$ & $\begin{array}{l}\text { TG, HDL-C, LDL- } \\
\text { C,TC }\end{array}$ & T2DM \\
\hline 9 & Akinci $^{42}$ & Turkey & $74(31 / 43)$ & $28.26 \pm 10.33 / 18.26 \pm 7.42$ & $12.9 \% / 44.2 \%$ & $\begin{array}{l}\text { cross-sectional } \\
\text { study }\end{array}$ & $\begin{array}{l}\text { TC, TG,LDL-C, } \\
\text { HDL-C, }\end{array}$ & DM \\
\hline 10 & Akbar $^{25}$ & India & $202(62 / 140)$ & $55.7 \pm 10.0 / 51.3 \pm 10.8$ & $33 \% / 61 \%$ & $\begin{array}{l}\text { cross-sectional } \\
\text { cohort }\end{array}$ & $\begin{array}{l}\text { TC,HDL,TG,TyG } \\
\text { index }\end{array}$ & T2DM \\
\hline 11 & Aktaş $^{26}$ & Turkey & $50(27 / 23)$ & $56.85 \pm 9.87 / 55.39 \pm 8.84$ & $7 \% / 10 \%$ & $\begin{array}{l}\text { cross-sectional } \\
\text { cohort }\end{array}$ & LDL,HDL,TC & DM \\
\hline 12 & Hwang ${ }^{23}$ & Korea & $530(239 / 291)$ & $20-80$ & $58.5 \% / 51.2 \% / 64.4 \% / 44.3 \%$ & $\begin{array}{l}\text { retrospective } \\
\text { study }\end{array}$ & $\begin{array}{l}\text { TC, TG,LDL-C, } \\
\text { HDL-C, }\end{array}$ & T2DM \\
\hline 13 & $\operatorname{Jane}^{43}$ & China & 628 & $\begin{array}{l}<6522.9 \% / 28.1 \% ; \geq 65 \\
77.1 \% / 71.9 \%\end{array}$ & $37 \% / 46.1 \%$ & $\begin{array}{l}\text { cross-sectional } \\
\text { study }\end{array}$ & TC, TG & T2DM \\
\hline 14 & Najaf $^{44}$ & Iran & 192 & $58.8 \pm 8.2 / 57.9 \pm 8.8$ & $48.9 \% 44.1 \%$ & $\begin{array}{l}\text { cross-sectional } \\
\text { study }\end{array}$ & $\begin{array}{l}\text { TG,TC,LDL- } \\
\text { C,HDL-C }\end{array}$ & T2DM \\
\hline 15 & Zoppini $^{45}$ & Italy & $557(461 / 96)$ & $58 \pm 9.6 / 56.7 \pm 6.6 / 57.8 \pm 9.8$ & $68.75 \% / 66.16 \%$ & A cohort & $\begin{array}{l}\text { LDL-C, HDL-C, } \\
\text { TGs }\end{array}$ & T2DM \\
\hline 16 & Ishibashi ${ }^{46}$ & Iapan & $107(78 / 28)$ & $\begin{array}{l}55.7 \pm 1.5 / 54.8 \pm 2.2 / 58.7 \pm 2 . \\
2 / 48.1 \pm 2.2\end{array}$ & $54.55 \% / 65.22 \%$ & $\begin{array}{l}\text { cross-sectional } \\
\text { cohort }\end{array}$ & $\begin{array}{l}\text { LDL-C,HDL- } \\
\text { C,TG }\end{array}$ & T2DM \\
\hline 17 & $\mathrm{Cho}^{47}$ & Korea & $48(15 / 33)$ & $\begin{array}{l}2006(55.93 \pm 9.23 / 54.26 \pm 1 \\
0.55) ; 2012(66.60 \pm 5.47 / 63 \\
15 \pm 8.81)\end{array}$ & NA & $\begin{array}{l}\text { retrospective } \\
\text { study }\end{array}$ & $\begin{array}{l}\text { TC, TG,LDL-C, } \\
\text { HDL-C, }\end{array}$ & T2DM \\
\hline 18 & $\begin{array}{l}\text { Rosales-Her- } \\
\text { nandez }{ }^{48}\end{array}$ & Canada & $82(60 / 12)$ & $\begin{array}{l}61.1 \pm 10.0 / 59.8 \pm 10.3 / 53 \\
6 \pm 14.4\end{array}$ & $69 \% / 64 \% / 50 \%$ & $\begin{array}{l}\text { a case control } \\
\text { study }\end{array}$ & $\begin{array}{l}\text { TC, TG,LDL-C, } \\
\text { HDL-C, }\end{array}$ & $\mathrm{DM}$ \\
\hline 19 & Jende $^{49}$ & Germany & $120(84 / 36)$ & $60.83 \pm 1.61 / 62.55 \pm 1.29$ & $60 \%$ & $\begin{array}{l}\text { cross-sectional } \\
\text { cohort }\end{array}$ & $\begin{array}{l}\text { TC, TG,LDL-C, } \\
\text { HDL-C, }\end{array}$ & $\begin{array}{l}\text { T1DM and } \\
\text { T2DM }\end{array}$ \\
\hline 20 & Smith $^{22}$ & USA & 218 & $58.5 \pm 9.1 / 60 \pm 7.9 / 57.5 \pm 9.8$ & $50 \% / 53 \% / 55 \%$ & $\begin{array}{l}\text { retrospective } \\
\text { study }\end{array}$ & TC,LDL,HDL & T2DM \\
\hline 21 & Katulanda ${ }^{50}$ & Sri Lanka & 528 & $55.0 \pm 12.4$ & 0.373 & $\begin{array}{l}\text { a case control } \\
\text { study }\end{array}$ & $\begin{array}{l}\text { TC, TG,LDL-C, } \\
\text { HDL-C, }\end{array}$ & $\begin{array}{l}\text { T1DM and } \\
\text { T2DM }\end{array}$ \\
\hline 22 & $\mathrm{Hsu}^{24}$ & China & $326(85 / 241)$ & $65.7 \pm 9.3 / 62.8 \pm 9.5$ & $38.8 \% / 31.1 \%$ & A cohort & TC, TG & T2DM \\
\hline 23 & Hsiao $^{51}$ & China & 271 & $55.33 \pm 11.04 / 56.49 \pm 6.94$ & $57.93 \% / 60 \%$ & $\begin{array}{l}\text { cross-sectional } \\
\text { study }\end{array}$ & TC & T2DM \\
\hline 24 & Ylitalo ${ }^{52}$ & USA & $100(9 / 91)$ & $63.2 \pm 0.7 / 55.8 \pm 0.4$ & $65.5 \% / 46.2 \%$ & A cohort & $\begin{array}{l}\text { TG,LDL-C, } \\
\text { HDL-C, }\end{array}$ & DM \\
\hline 25 & Spallone $\mathrm{e}^{53}$ & Italy & $191(135 / 56)$ & $\begin{array}{l}59.9 \pm 9.7 / 57.2 \pm 10.5 / 58 . \\
1 \pm 9.6\end{array}$ & $44.87 \% / 71.93 \% / 57.14 \%$ & $\begin{array}{l}\text { a case control } \\
\text { study }\end{array}$ & $\begin{array}{l}\text { TC, TG,LDL-C, } \\
\text { HDL-C, }\end{array}$ & $\begin{array}{l}\text { T1DM and } \\
\text { T2DM }\end{array}$ \\
\hline 26 & Terekeci ${ }^{65}$ & USA & $42(25 / 17)$ & $58.80 \pm 8.60 / 55.18 \pm 6.41$ & $50 \%$ & A cohort & TC,TG & T2DM \\
\hline 27 & Faisal $^{27}$ & Bahrain & $1225(526 / 689)$ & $54 / 52$ & $42.99 \%$ & $\begin{array}{l}\text { cross-sectional } \\
\text { cohort }\end{array}$ & TC,TG,HDL-C & $\begin{array}{l}\text { T1DM and } \\
\text { T2DM }\end{array}$ \\
\hline 28 & Coppini $^{55}$ & UK & $300(100 / 200)$ & $66.2 \pm 9.4 / 49.2 \pm 16.3$ & Gender (M:F) $1.3: 1 / 2: 1$ & $\begin{array}{l}\text { a retrospective } \\
\text { case-control } \\
\text { study }\end{array}$ & $\begin{array}{l}\text { TC, TG,LDL-C, } \\
\text { HDL-C, }\end{array}$ & DM \\
\hline 29 & Kempler $^{28}$ & $\begin{array}{l}31 \text { centres in } \\
\text { 16 European } \\
\text { countries }\end{array}$ & 3270 & $32.7 \pm 10.2$ & $51.62 \%$ & A cohort & $\begin{array}{l}\text { TC, TG,LDL-C, } \\
\text { HDL-C, }\end{array}$ & TIDM \\
\hline 30 & Christen $^{64}$ & USA & 407 & $31.4 \pm 1.4$ & $75.30 \%$ & $\begin{array}{l}\text { a prospective } \\
\text { cohort study }\end{array}$ & LDL,HDL,TG & T1DM \\
\hline 31 & Maser $^{57}$ & Pittsburgh & $363(228 / 135)$ & $34 \pm 6 / 28 \pm 6$ & $52 \% / 50 \%$ & A cohort & LDL,HDL,TG & T1DM \\
\hline 32 & Maser $^{58}$ & Pittsburgh & $168(105 / 63)$ & $30 \pm 3 / 29 \pm 3$ & $49 \% / 57 \%$ & A cohort & LDL,HDL,TG & T1DM \\
\hline 33 & Orchard $^{59}$ & Pittsburgh & $325(57 / 268)$ & $<17$ & $47 \% / 56 \%$ & A cohort & $\begin{array}{l}\text { TC,TG,LDL-C, } \\
\text { HDL-C, }\end{array}$ & T1DM \\
\hline 34 & Tesfaye $^{18}$ & $\begin{array}{l}31 \text { centres in } \\
16 \text { European } \\
\text { countries }\end{array}$ & 3250 & NA & $51.32 \%$ & A cohort & LDL,HDL,VLDL & TIDM \\
\hline \multicolumn{9}{|c|}{ Continued } \\
\hline
\end{tabular}




\begin{tabular}{|c|c|c|c|c|c|c|c|c|}
\hline Number & References & Country & $\begin{array}{l}\mathrm{N} \text { (case/ } \\
\text { control) }\end{array}$ & Age & M\% & Study design & $\begin{array}{l}\text { Outcome } \\
\text { reported }\end{array}$ & Diabetes \\
\hline 35 & Simmons $^{60}$ & UK & $33(20 / 13)$ & $49 \pm 5 / 50 \pm 11 / 50 \pm 6$ & $63.64 \% / 66.67 \% / 46.15 \%$ & $\begin{array}{l}\text { cross-sectional } \\
\text { cohort }\end{array}$ & TC, TG & T1DM \\
\hline 36 & Tesfaye $^{9}$ & $\begin{array}{l}31 \text { centers in } \\
\text { the European } \\
\text { Diabetes }\end{array}$ & $1172(276 / 896)$ & $29.8 \pm 8.1 / 33.6 \pm 10.0$ & $51.1 \% / 48.6 \%$ & $\begin{array}{l}\text { prospective } \\
\text { study }\end{array}$ & $\begin{array}{l}\text { TC, TG,LDL-C, } \\
\text { HDL-C, }\end{array}$ & T1DM \\
\hline 37 & Witte $^{56}$ & UK & $956(163 / 793)$ & $34.5 \pm 10.3 / 30.7 \pm 8.4$ & $35.58 \% / 6.68 \%$ & A cohort & $\begin{array}{l}\text { TC, TG,LDL, } \\
\text { HDL }\end{array}$ & T1DM \\
\hline 38 & Callaghan $^{62}$ & USA & 2382 & $73.5 \pm 2.9$ & $48.30 \%$ & A cohort & TG,HDL & $\mathrm{DM}$ \\
\hline 39 & Callaghan $^{63}$ & China & 4002 & $51.6 \pm 11.8$ & $51 \%$ & $\begin{array}{l}\text { A cross-sec- } \\
\text { tional }\end{array}$ & TG,HDL & $\mathrm{DM}$ \\
\hline
\end{tabular}

Table 1. Studies included in the meta-analysis.

\begin{tabular}{|c|c|c|c|c|c|c|}
\hline Number & References & Selection & Comparability & \begin{tabular}{|l|} 
Outcome \\
ascertainment
\end{tabular} & $\begin{array}{l}\text { Bias risk (Total } \\
\text { scores) }\end{array}$ & $\begin{array}{l}\text { Final quality } \\
\text { conclusion }\end{array}$ \\
\hline 1 & Jende $^{35}$ & 4 & 1 & 1 & 6 & Fair \\
\hline 2 & Hosny $^{36}$ & 4 & 2 & 1 & 7 & Good \\
\hline 3 & Song $^{29}$ & 3 & 2 & 1 & 6 & Fair \\
\hline 4 & Vural $^{37}$ & 2 & 2 & 1 & 5 & Fair \\
\hline 5 & Andersen $^{38}$ & 3 & 2 & 3 & 8 & Good \\
\hline 6 & Mizokami-Stout $^{39}$ & 3 & 2 & 3 & 8 & Good \\
\hline 7 & Litzelman $^{40}$ & 4 & 2 & 2 & 8 & Good \\
\hline 8 & Aryan $^{41}$ & 3 & 2 & 2 & 7 & Good \\
\hline 9 & Akinci $^{42}$ & 2 & 2 & 1 & 5 & Fair \\
\hline 10 & $\mathrm{Akbar}^{25}$ & 3 & 1 & 2 & 6 & Fair \\
\hline 11 & Aktaş $^{26}$ & 2 & 1 & 2 & 5 & Fair \\
\hline 12 & Hwang $^{23}$ & 4 & 2 & 1 & 7 & Good \\
\hline 13 & Jane $^{43}$ & 4 & 1 & 3 & 8 & Good \\
\hline 14 & Najafi $^{44}$ & 3 & 1 & 2 & 6 & Fair \\
\hline 15 & Zoppini $^{45}$ & 4 & 2 & 3 & 9 & Good \\
\hline 16 & Ishibashi $^{46}$ & 2 & 1 & 2 & 5 & Fair \\
\hline 17 & $\mathrm{Cho}^{47}$ & 2 & 1 & 2 & 5 & Fair \\
\hline 18 & Rosales-Hernandez ${ }^{48}$ & 3 & 1 & 1 & 5 & Fair \\
\hline 19 & Jende $^{49}$ & 3 & 2 & 1 & 6 & Fair \\
\hline 20 & Smith $^{22}$ & 3 & 2 & 2 & 7 & Good \\
\hline 21 & Katulanda $^{50}$ & 4 & 2 & 2 & 8 & Good \\
\hline 22 & $\mathrm{Hsu}^{24}$ & 4 & 2 & 1 & 7 & Good \\
\hline 23 & $\mathrm{Hsiao}^{51}$ & 4 & 3 & 1 & 8 & Good \\
\hline 24 & Ylitalo $^{52}$ & 3 & 2 & 1 & 6 & Fair \\
\hline 25 & Spallone $^{53}$ & 3 & 1 & 2 & 6 & Fair \\
\hline 26 & Terekeci $^{65}$ & 2 & 2 & 1 & 5 & Fair \\
\hline 27 & Faisal $^{27}$ & 4 & 2 & 3 & 9 & Good \\
\hline 28 & Coppini $^{55}$ & 3 & 2 & 3 & 8 & Good \\
\hline 29 & Kempler $^{28}$ & 4 & 2 & 3 & 9 & Good \\
\hline 30 & Christen $^{64}$ & 4 & 1 & 1 & 6 & Fair \\
\hline 31 & Maser $^{57}$ & 3 & 2 & 2 & 7 & Good \\
\hline 32 & Maser $^{58}$ & 3 & 1 & 1 & 5 & Fair \\
\hline 33 & Orchard $^{59}$ & 3 & 1 & 2 & 6 & Fair \\
\hline 34 & Tesfaye $^{18}$ & 4 & 2 & 3 & 9 & Good \\
\hline 35 & Simmons $^{60}$ & 3 & 1 & 1 & 5 & Fair \\
\hline 36 & Tesfaye $^{9}$ & 4 & 2 & 3 & 9 & Good \\
\hline 37 & Witte $^{56}$ & 3 & 2 & 3 & 8 & Good \\
\hline 38 & Callaghan $^{62}$ & 4 & 2 & 3 & 9 & Good \\
\hline 39 & Callaghan $^{63}$ & 4 & 2 & 3 & 9 & Good \\
\hline
\end{tabular}

Table 2. Risk of bias analysis in each study. 
A

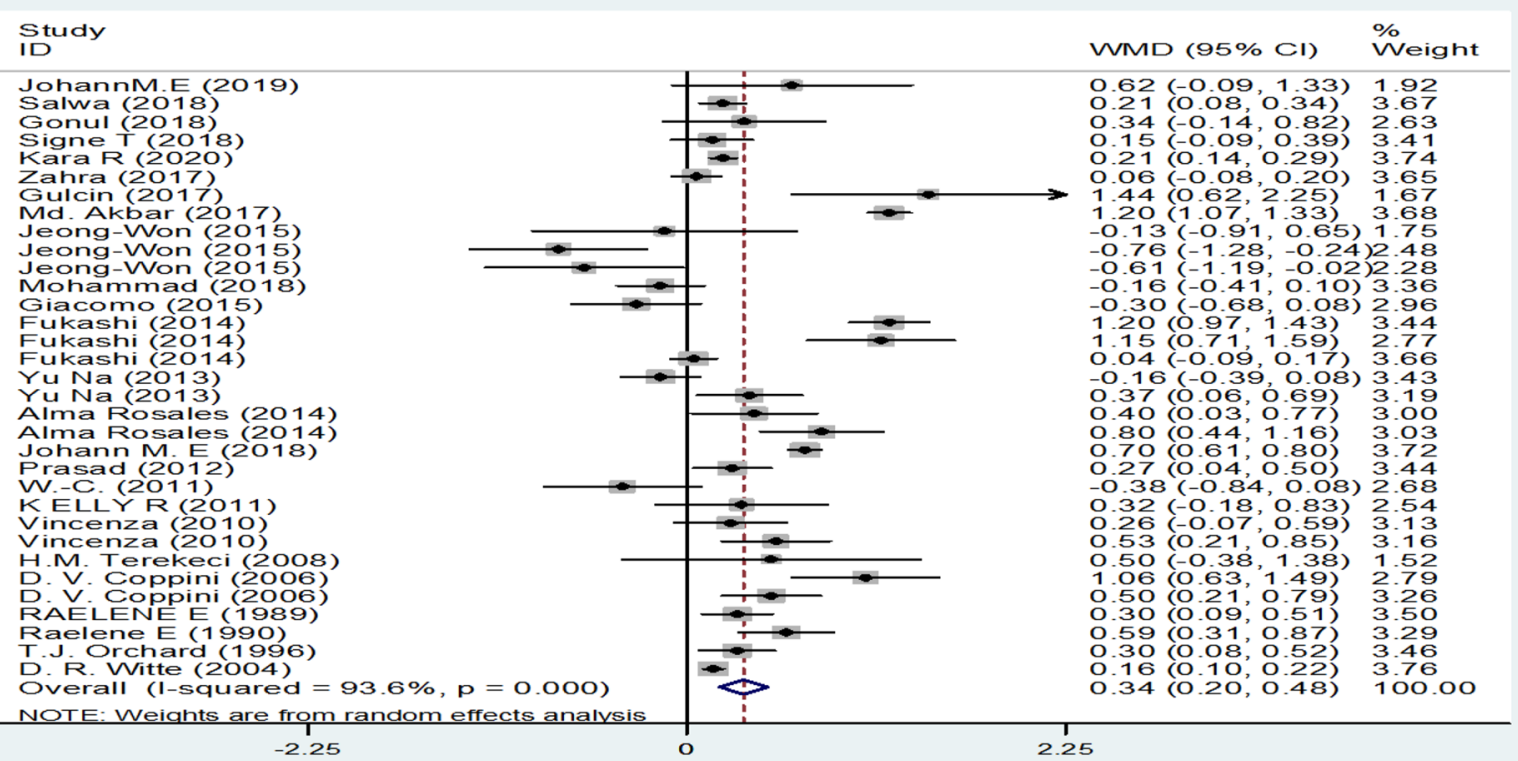

B

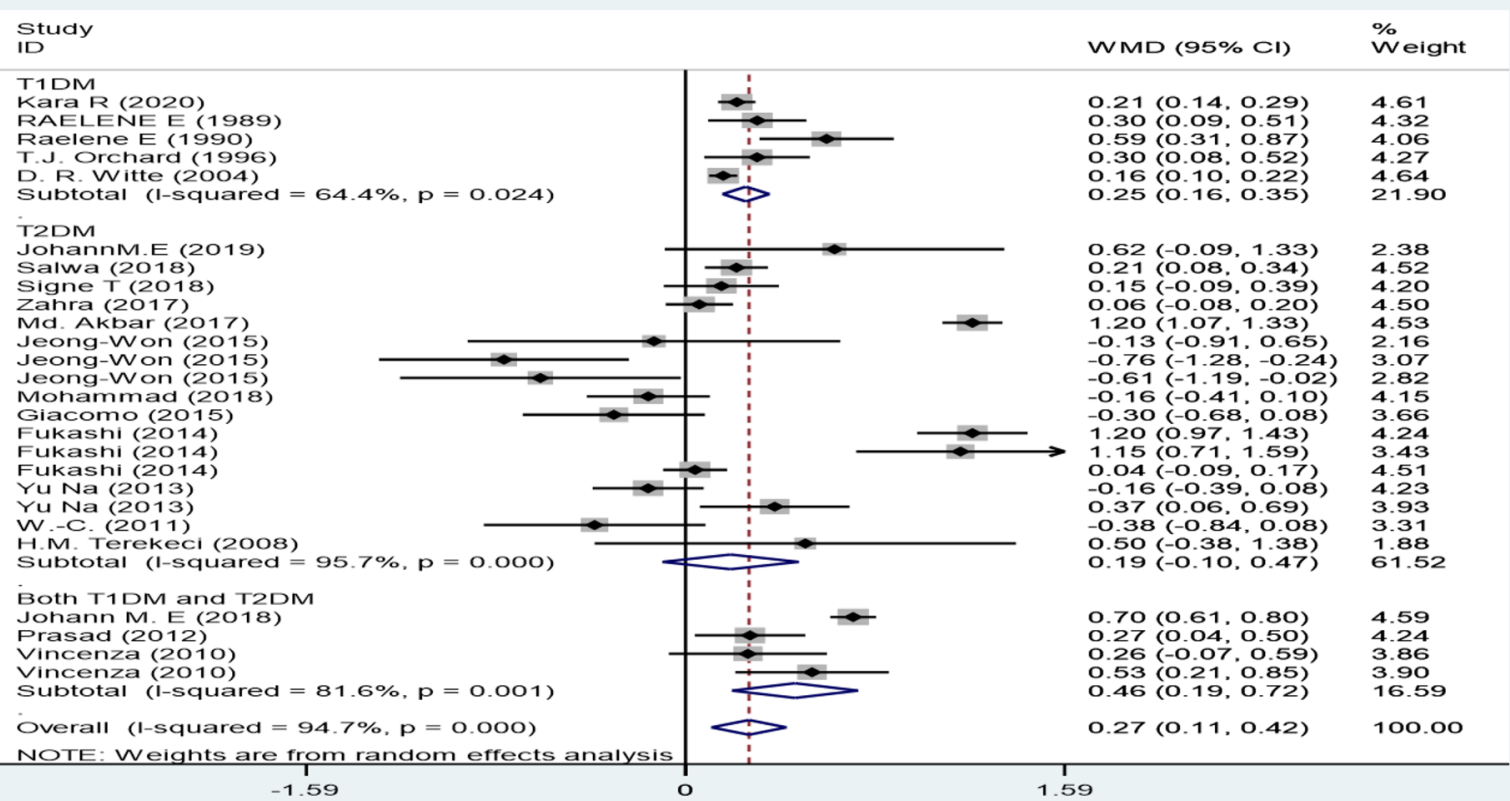

Figure 2. TG levels (A) in people with DN versus those without DN. TG levels in the subgroup analysis stratified by the type of diabetes (B) and symptom severity (C). OR (D) for DN in patients according to serum TG levels. 
C

Study

ID

WMD $(95 \%$ CI) Weight

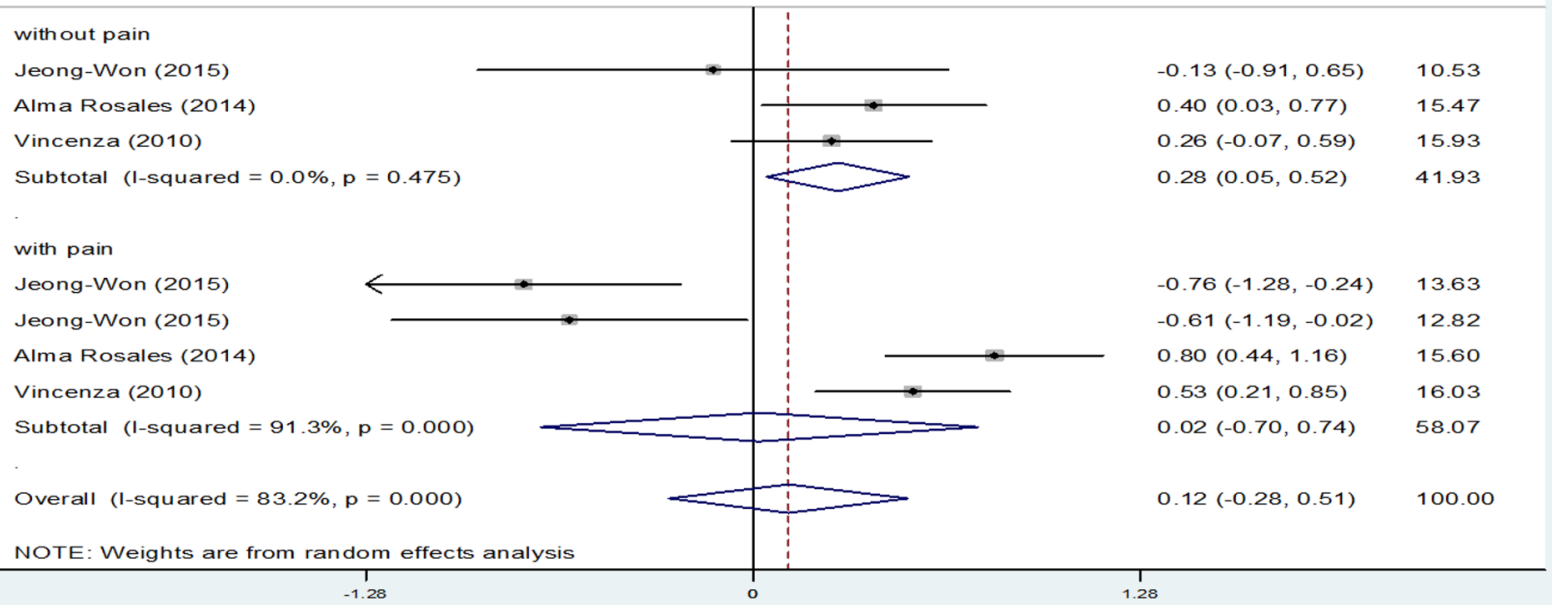

D

Study

ID

1.28

$\%$

Weight

8.42

4.70

3.26

4.58

8.20

6.23

6.15

6.19

2.24

2.63

2.66

6.15

6.33

6.52

8.74

8.97

8.03

100.00

Overall $(1$-squared $=86.1 \%, p=0.000)$

NOTE: Weights are from random effects analysis 229

4.36

Figure 2. (continued)

Serum TG levels between DN and non-DN patients/healthy controls. The pooled TG results with of 35 studies on TG showed a significantly increased serum TG levels in DN patients compared to non-DN patients with a random-effects model (MD (95\% CI): $\left.0.34(0.20-0.48), I^{2}=93.6 \%, \mathrm{p}<0.001\right)$ (Fig. 2A). Moreover, the serum TG levels of neuropathy patients with T1DM were higher than those of control patients (Fig. 2B). Patients with moderate or severe pain had no significant difference in TG levels of TG than compared with patients with mild or painless controls patients (MD (95\% CI): $\left.0.12(-0.28-0.51), I^{2}=83.2 \%, \mathrm{p}<0.001\right)$ (Fig. 2C). Furthermore, compared with the lower serum TG level category, the highest serum TG level showed an increased the risk of DN (OR (95\% CI): $\left.1.36(1.20-1.54), I^{2}=86.1 \%, \mathrm{p}<0.001\right)$ (Fig. 2D).

Serum TC between DN and non-DN patients. Twenty-nine studies, provided data on TC. Forest plot indicated that the expression level of TC in DN patients was not significantly different from that in control patients with a random-effects model (MD (95\% CI): $\left.-0.03(-0.14-0.09), I^{2}=82.9 \% ; \mathrm{p}<0.001\right)$ (Fig. $\left.3 \mathrm{~A}\right)$. The expression levels of TC in both T1DM neuropathy and T2DM neuropathy were not significantly different from those in the control group (Fig. 3B). Additionally, for symptomatic DN, moderate or severe DN patients had lower levels of serum TC compared to mild or painless DN patients (MD (95\% CI): $-0.31(-0.49--0.31), I^{2}=0 \%$, $\mathrm{p}=0.991)$ (Fig. 3C). Patients with high levels of TC level had no influence on the risk of DN (OR (95\% CI): 1.02 $(1.00-1.04)$, and there was obvious evidence of significant heterogeneity among studies $\left(I^{2}=84.7 \%, \mathrm{p}<0.001\right)$ (Fig. 3D). 
A

Study

ID

WMD (95\% CI)

$0.00(-0.08,0.08)$

$-0.58(-1.03,-0.13)$

$0.64(-1.035,0.93)$

$-0.04(-0.46,0.37)$

$-0.85(-1.52,-0.18)$

$-0.33(-0.79,0.14)$

$0.60(-0.04,1.24)$

$-0.10(-0.27,0.07)$

$0.74(0.19,1.28)$

$0.00(-0.35,0.35)$

$-0.30(-0.57,-0.03)$

$-0.33(-0.63,-0.02)$

$-0.39(-0.66,-0.12)$

$-0.07(-0.43,0.29)$

$0.18(-0.12,0.48)$

$0.18(-0.12,0.48)$

$-0.40(-0.91,0.11)$

$-0.30(-0.74,0.14)$

$0.27(0.21,0.33)$

$0.00(-0.46,0.46)$

$0.01(-0.27,0.29)$

$-0.27(-0.59,0.04)$

$0.25(-0.64,1.14)$

$0.50(0.24,0.76)$

$-0.20(-0.43,0.03)$

$0.14(-0.05,0.33)$

-0.03 (-0.14, 0.09 ) veight

5.98

3.28

4.51

3.55

2.08

3.18

2.22

5.45

2.70

4.03

4.68

4.39

4.39

4.66

3.95

4.41

2.89

3.36

6.06

3.19

4.58

4.32

4.32

1.39

4.81

4.99

5.35
100.00

Overall (I-squared $=82.9 \%, p=0.000$ )

NOTE: Weights are from random effects analysis

$-1.52$

o

152

B

Study

T1DM

Kara R (2020)

D. R. Witte (2004)

Subtotal (I-squared $=44.9 \%, p=0.178$ )

T2DM

JohannM.E (2019)

Salwa (2018)

Signe T (2018)

Zahra (2017)

Md. Akbar (2017)

Jeong-Won (2015)

Jeong-Won (2015)

Jeong-Won (2015)

Yu Na (2013)

Yu Na (2013)

A. Gordon (2013)

W. G. (2011)

H.M. Terekeci (2008)

Subtotal (I-squared $=73.6 \%, p=0.000)$

Both T1DM and T2DM

Johann M. E (2018)

Prasad (2012)

Subtotal ( 1 -squared $=67.6 \%, p=0.079$ )

Overall (I-squared $=84.5 \%, \mathrm{p}=0.000$ )

NOTE: Weights are from random effects analysis

$-1.52$

WMD (95\% CI)

$\%$

$0.00(-0.08,0.08) \quad 8.08$ $\begin{array}{ll}0.14(-0.05,0.33) & 7.17 \\ 0.04(-0.08,0.17) & 15.25\end{array}$

$-0.58(-1.03,-0.13) 4.27$ $0.64(0.35,0.93) \quad 5.97$

$-0.85(-1.52,-0.18) 2.66$

$-0.33(-0.79,0.14) 4.13$

$-0.10(-0.27,0.07) 7.31$

$\mathrm{O} .00(-0.35,0.35) \quad 5.30$

$-0.30(-0.57,-0.03) 6.21$

$-0.33(-0.63,-0.02) 5.80$

$-0.07(-0.43,0.29)$ ( 18.18

$0.18(-0.12,0.48) 5.83$

$0.00(-0.46,0.46), 4.15$

$0.27(-0.59,0.04) 5.71$

$0.25(-0.64,1.14) \quad 1.76$

$-0.14(-0.32,0.04) \quad 70.47$

$0.27(0.21,0.33) \quad 8.20$

$0.18(-0.07,0.42) \quad 14.28$

$-0.06(-0.20,0.07) 100.00$

.52

Figure 3. TC levels (A) in people with DN versus those without DN. TC levels in the subgroup analysis stratified by the type of diabetes (B) and symptom severity (C). OR (D) for DN in patients according to serum TC levels. 


\section{C}

Study

$\%$

ID

WMD $(95 \% \mathrm{Cl}) \quad$ Weight

without pain

Jeong-Won (2015)

Alma Rosales (2014)

Subtotal (I-squared $=38.4 \%, p=0.203$ )

with pain

Jeong-Won (2015)

Jeong-Won (2015)

Alma Rosales (2014)

Subtotal (I-squared $=0.0 \%, p=0.991$ )

Overall (I-squared $=0.0 \%, p=0.597$ )

NOTE: Weights are from random effects analysis

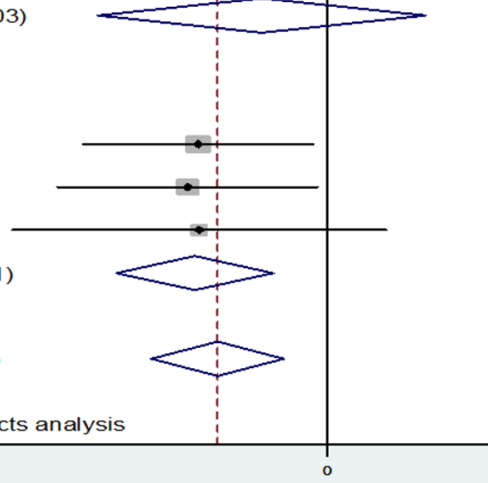

$0.00(-0.35,0.35)$

19.62

$-0.40(-0.91,0.11)$

9.20

$-0.15(-0.54,0.23) \quad 28.82$

$-0.30(-0.57,-0.03) \quad 32.95$

$-0.33(-0.63,-0.02) \quad 25.79$

$-0.30(-0.74,0.14) \quad 12.44$

$-0.31(-0.49,-0.13) \quad 71.18$

$-0.26(-0.41,-0.10) \quad 100.00$

D

Study

ID

Lige Song 2015 DEBRA K1997 DEBRA K1997 Sui-Whi 2016 J-YH SIAO 2011 P. Kempler 2002 P. Kempler 2002 P. Kempler 2002 William G 1999 William G 1999 William G 1999 S.Tesfaye 1996 S.Tesfaye 1996 S.Tesfaye 1996 Faisal 2007 Solomon 2005

Overall (I-squared $=84.7 \%, \mathrm{p}=0.000)$ NOTE: Weights are from random effects analysis 357
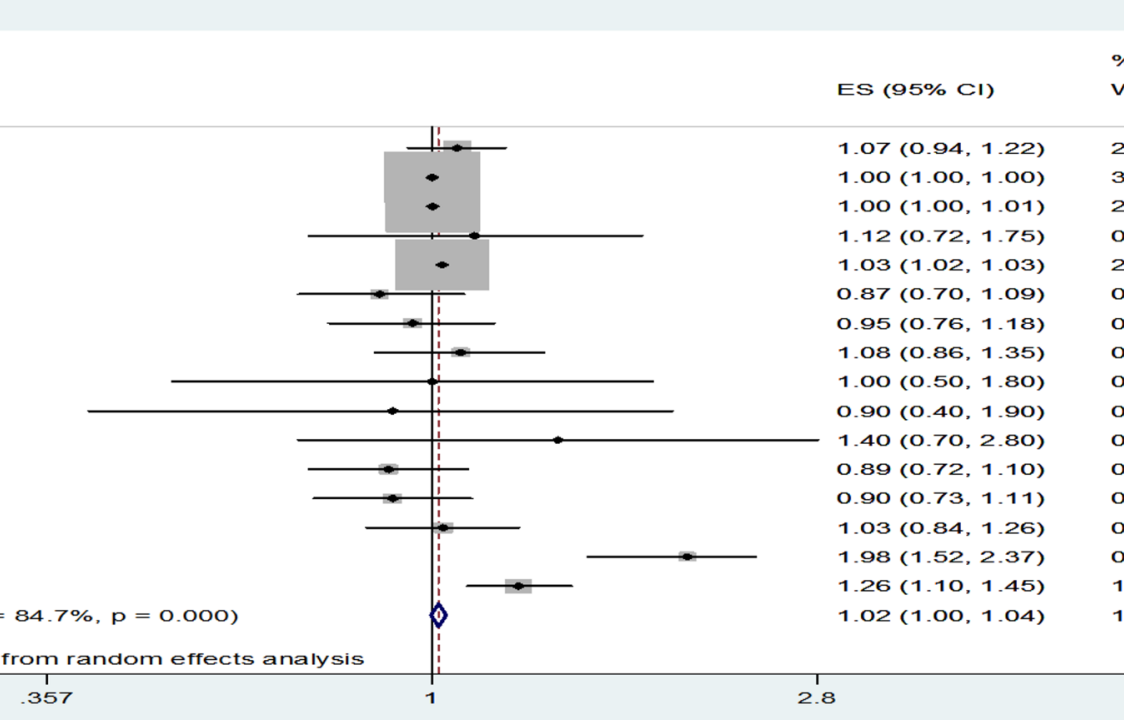

07 (0 94, 1,22$)$

$1.00(1.00,1.00)$

$1.00(1.00,1.01)$

$1.12(0.72,1.75)$

$1.03(1.02,1.03)$

$0.87(0.70,1.09)$

$0.95(0.76,1.18)$

$1.08(0.86,1.35)$

$1.00(0.50,1.80)$

$0.90(0.40,1.90)$

$1.40(0.70,2.80)$

$0.89(0.72,1.10)$

$0.90(0.73,1.11)$

$1.03(0.84,1.26)$

$1.98(1.52,2.37)$

$1.26(1.10,1.45)$

$1.02(1.00,1.04)$

Weight

2.18

30.34

29.87

0.19

29.63

0.77

0.78

0.74

0.09

0.06

0.08

0.84

0.85

0.91

0.76

1.90

100.00

Figure 3. (continued)

Serum HDL levels between of DN and non-DN patients/healthy controls. Data on HDL levels for DN were obtained from 34 studies. The results showed that serum HDL levels in patients with DN were lower than those in the control group with a random-effects model (MD (95\% CI): $-0.05\left(-0.08--0.02, I^{2}=81.3 \%\right.$; $\mathrm{p}<0.001$ ) (Fig. 4A). Interestingly, subgroup analysis found that only patients with T1DM neuropathy had lower HDL levels than patients in the control group, while serum HDL levels in patients with T2DM were not different from those in the control group (Fig. 4B). The change in the MD for serum HDL levels was not significantly different between DPN and painless neuropathy (MD (95\% CI): $-0.07(-0.04-0.01), I^{2}=58.8 \%, p=0.013$ (Fig. 4C). In addition, high levels of HDL were observed to decrease the risk of DN (OR (95\% CI):0.85 (0.75-0.96), $I^{2}=72.6 \%, \mathrm{p}<0.001$ (Fig. 4D).

Serum LDL levels between DN and non-DN patients/healthy controls. Twenty-nine trials showed no effect of LDL levels on DN. DN patients showed no difference in LDL levels compared to the control group with the random-effects model (MD (95\% CI): $-0.00\left(-0.08-0.08, I^{2}=78.9 \%\right.$; $\left.<<0.001\right)$ (Fig. 5A). There was no significant difference in LDL levels in either type 1 or type 2 diabetic neuropathy patients compared with the control group (Fig. 5B). Moderate or severe pain DN patients had lower levels of LDL compared to than mild 
A

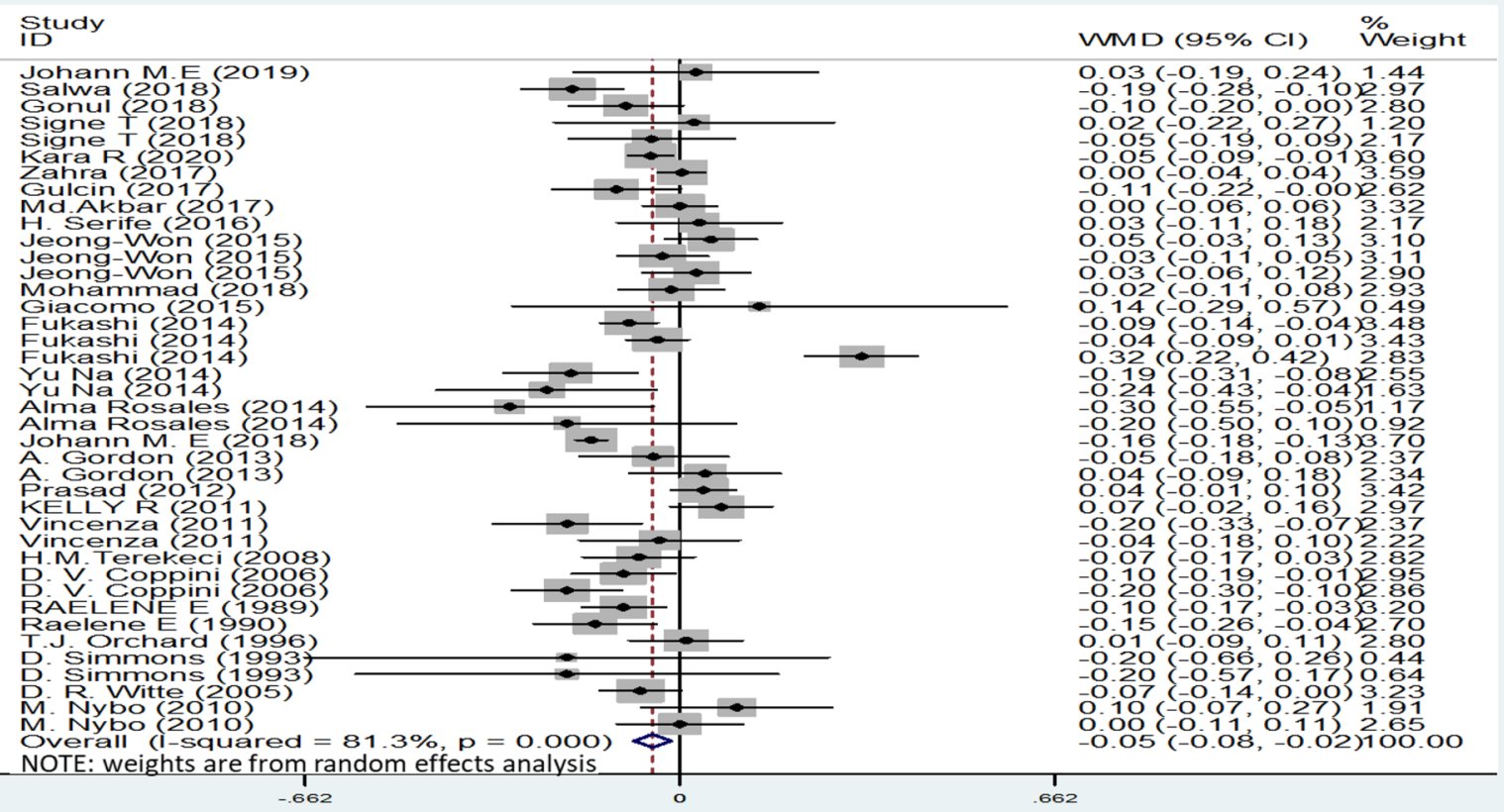

B

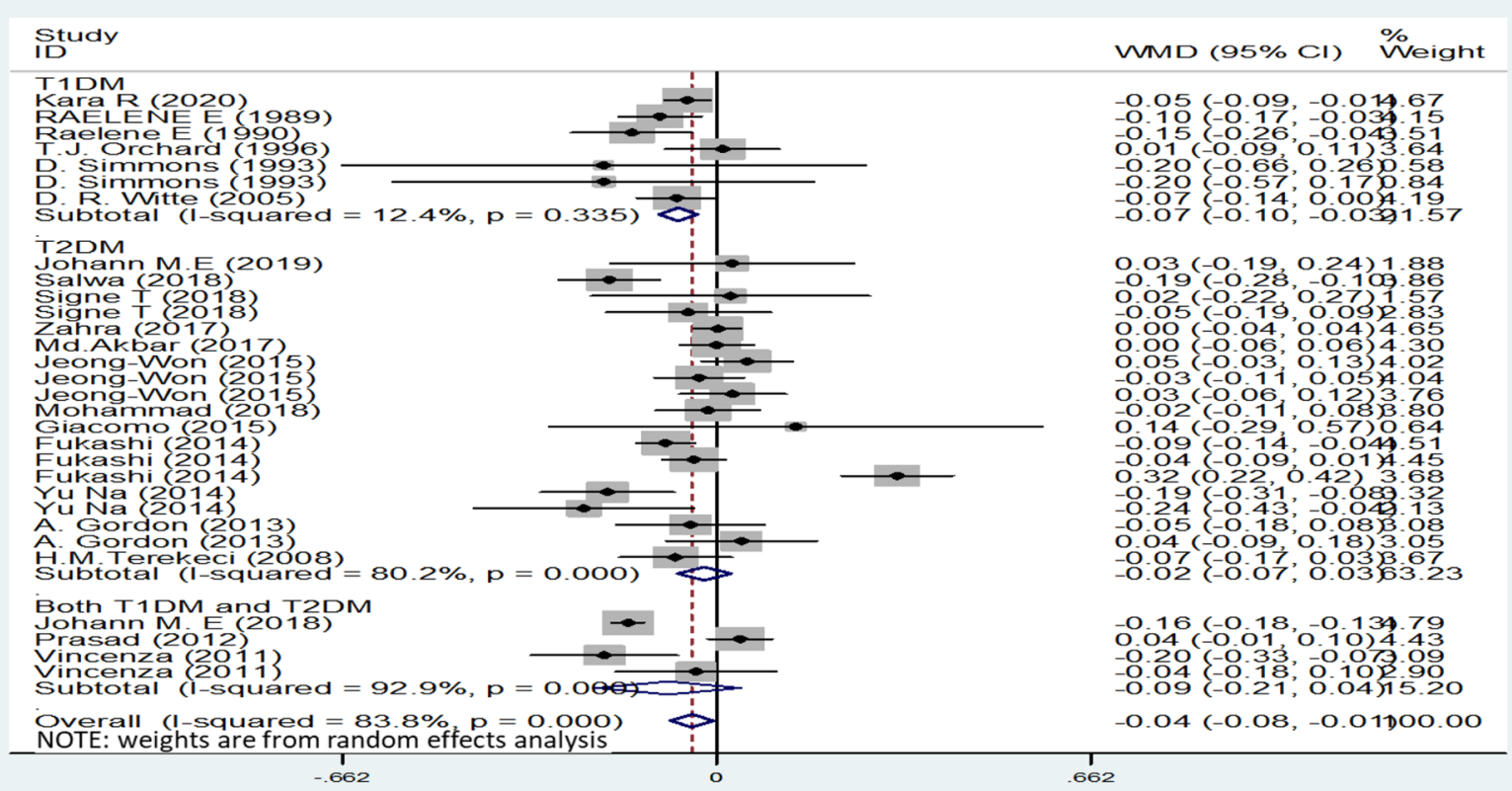

Figure 4. HDL levels (A) in people with DN versus those without DN. HDL levels in the subgroup analysis stratified by the type of diabetes (B) and symptom severity (C). OR (D) for DN in patients according to serum HDL levels. 
C

Study

ID

$\%$

without pain

without pain

Jeong-Won (2015)

Alma Rosales (2014)

Vincenza (2011)

D. Simmons (1993)

Subtotal $($ I-squared $=76.3 \%, p=0.005)$

with pain

Jeong-Won (2015)

Jeong-Won (2015)

Alma Rosales (2014)

Vincenza (2011)

D. Simmons (1993)

Subtotal $(\mathrm{I}$-squared $=40.8 \%, \mathrm{p}=0.149$ )

Overall (I-squared $=58.8 \%, p=0.013$ )

NOTE: Weights are from random effects analysis

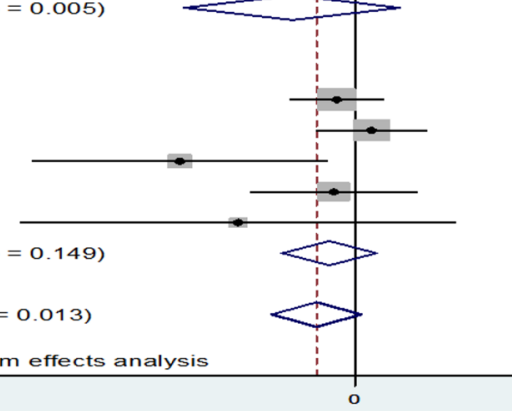

WMD $(95 \% \mathrm{Cl})$

Weight

$0.05(-0.03,0.13) \quad 18.80$

-0.20 (-0.50, 0.10) 5.15

$-0.20(-0.33,-0.07) \quad 14.03$

$-0.20(-0.66,0.26) \quad 2.44$

$-0.11(-0.29,0.08) \quad 40.42$

$-0.03(-0.11,0.05) \quad 18.87$

$0.03(-0.06,0.12) \quad 17.44$

$-0.30(-0.55,-0.05) \quad 6.62$

$-0.04(-0.18,0.10) \quad 13.07$

$-0.20(-0.57,0.17) \quad 3.58$

$-0.04(-0.12,0.04) \quad 59.58$

$-0.07(-0.14,0.01) \quad 100.00$

D

study

ID

Lige Song 2015

DEBRA K1997

DEBRA K1997

P. Kempler 2002

P. Kempler 2002

P. Kempler 2002

William G 1999

William 61999

William G 1999

S.Tesfaye 1996

S.Tesfaye 1996

S.Tesfaye 1996

Brian C 2016

Brian C 2017

Overall (1-squared $=72.6 \%, p=0.000)$

NOTE: Weights are from random effects analysis

4

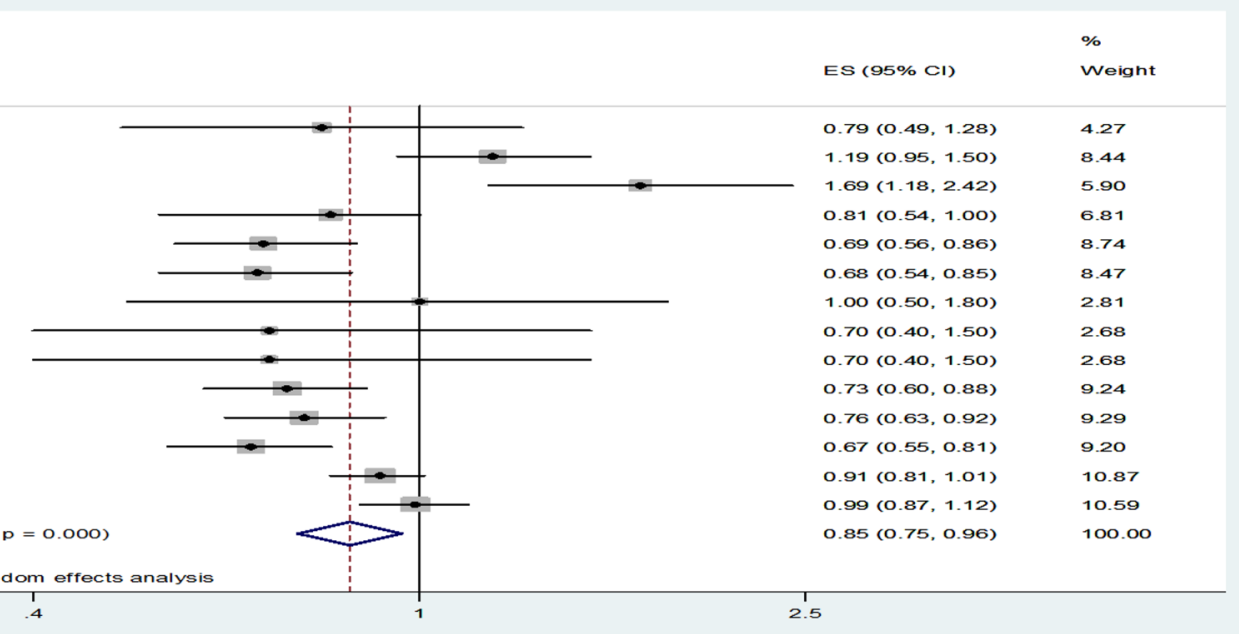

Figure 4. (continued)

or painless DN patients (MD (95\% CI): $\left.-0.19(-0.32--0.06), I^{2}=0 \%, \mathrm{p}=0.705\right)$ (Fig. $\left.5 \mathrm{C}\right)$. Serum LDL levels increased the risk of DN (OR (95\% CI): $1.10\left(1.02-1.19, I^{2}=17.8 \%, \mathrm{p}=0.274\right)$ (Fig. 5D).

Sensitivity analyses and publication bias. Visual inspection of the funnel plot did not reveal remarkable asymmetry (Figs. 6, 7). Sensitivity analysis was performed to analyse the pooled results of the remaining studies by sequential removal of individual studies. There was no significant change in the overall outcomes after removing any single study, suggesting the stability and reliability of our results and that the data were not influenced by any given study (Figs. 8, 9). Thus, the above results suggest that publication bias was not apparent in this meta-analysis.

\section{Discussion}

$\mathrm{DN}$ is a common cause of morbidity and death among patients with diabetes ${ }^{66}$; this form of neuropathy is characterized by pain, paraesthesia, sensory loss, an increased frequency of falls, and reduced quality of life (QOL) ${ }^{9,67}$. It is obvious that DN poses a heavy health challenge to individuals. The duration and level of hyperglycaemia are important determinants of diabetic complications, including $\mathrm{DN}^{68}$. The risk of $\mathrm{DN}$ can be reduced with intensive blood glucose control in T1DM patients. Intensive blood glucose control has little influence in patients with $\mathrm{T}_{2} \mathrm{DM}^{15-17}$. One study showed that DN remained substantial, despite intensive control of the glucose level ${ }^{68}$. Moreover, except for optimal glycaemic control, there have been no definite positive prevention studies of other risk factor modifications for DN. Thus, there may be risk factors aside from hyperglycaemia involved in the development of DN. Identifying them, particularly if they are modifiable, might lead to new risk-reduction strategies. Accumulated evidence has shown a correlation between DN and serum lipid profiles but has shown 
A

Study

Johann M.E.(2019)

Salva (2018)

Signe ${ }^{2}(2018)$

Kara R (2020)

Zahra (2017)

$H$ Serife $(2016)$
Jeong

Jeong-Won (2015)

Jeong-Won (2015)

Mohammad(2018)

Giacomo(2015)

Fukashi(2014)

Fukashi(2O14)

Yu Na (2013)

Alma Rosales(2014)

Alma Rosales(2014)

A. Gordon (2O13)

Prasad (2012)

KELLYR (2011)

Vincenza(2010)

H.M.Terekeci 2008

Raelene (1990)

T.J. Orchard (1996)

D. Simmons (1993)

D. R. Vitte (2OO4)

NOTE: Weights are from random effects analys

$-2.14$

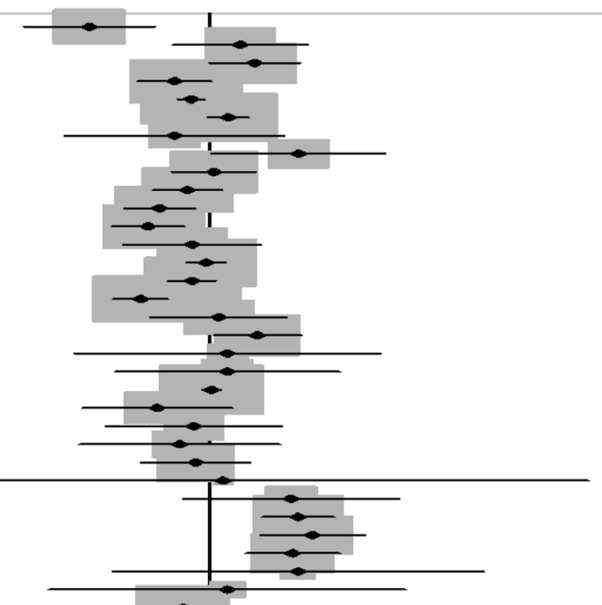

WMD $(95 \%$ Cl) \% Weight

$-0.68(-1.04,-0.32) 2.59$

$0.17(-0.20,0.55) \quad 2.50$

0.20 (-0.40, 0.00$) 4.13$

-0.20 (-0.40, 0.00) 4.13

$0.10(-0.01,0.21) 5.04$

$0.50(0.021,0.99) \quad 1.83$

$0.12(-0.31,0.06) 4.25$

0.35 (-0.55, -0.15)4.15

$0.10(-0.48,0.28) 2.4$

$-0.10(-0.23,0.03) 4.87$

$-0.39(-0.54,-0.24) 4.67$

$0.27(0.03,0.51) \quad 3.69$

0.10 (-0.53, 0.73) 1.26

$-0.30(-0.71,0.12) 2.21$

$0.17(-0.73,0.39) 1.49$

$0.08(-0.38,0.22) 3.11$

$0.08(-1.99,2.14) \quad 015$

$0.50(0.30,0.70) 4.16$

$0.58(0.29,0.87) \quad 3.22$

$0.50(-0.54,1.54) \quad 0.53$

$0.10(-0.91,1,11) \quad 0.57$

0.00 (-0.08,0.08) 10000

o

2.14

B
Study
WMD $(95 \%$ CI)
\% Weight

\section{T1DM}

Kara $(2020)$

RAELENE E (1989)

Raelene (1990)

T.J. Orchard (1996)

D. Simmons (1993)

D. Simmons (1993)

Subtotal (I-squar

T2DM

Johann M.E.(2019)

Signe 1 (2018)

Zahra (2017)

Jeong-Won(2015)

Jeong-Won(2015)

Jeong-Won(2015)

Giacomo(2015)

Fukashi(2014)

Fukashi(2014)

Yu Na (2O13)

A. Gordon (2013)
Sibtotal (I-squared $=78.0 \%, p=0.000)<$

Both T1DM and T2DM

Johann M.E.(2018)

Vincenza(2010)

Vincenza(2010)

Subtotal (I-squared $=0.0 \%, p=0.920)$

Overall (1-squared $=82.1 \%, \mathrm{p}=0.000$ )

NOTE: Weights are from random effects analysis

$\begin{array}{lll}\text {-2.14 } & 0 & 2.14\end{array}$

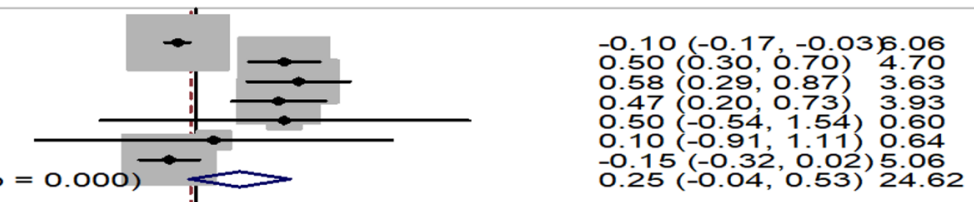

$-0.10(-0.17,-0.03) 6.06$ .58 (0.29, 0.87) 3.63 $0.50(-0.54,1.54) 0.60$ $-0.15(-0.32,0.02) 5.06$

$-0.68(-1.04,-0.32) 2.92$ $-0.20(-0.40,0.00) 4.67$ $0.10(-0.01,0.21) 5.71$ $0.02(-0.21,0.25) 4.32$ $-0.12(-0.31,0.06) 4.80$ -0.28 (-0.48, -0.09)4.75 $-0.10(-0.48,0.28) 2.78$ $-0.02(-0.13,0.09) 5.75$ $-0.39(-0.54,-0.24) 5.29$ $0.05(-0.33,0.43) 2.80$ $-0.30(-0.71,0.12) 2.50$

$0.01(-0.04,0.06) 6.20$ $-0.09(-0.58,0.40) 2.01$ $0.08(-0.38,0.22) 3.51$ $0.08(-1.99,2.14) 0.17$ $-0.03(-0.11,0.06) 100.00$

\section{4}

Figure 5. LDL levels (A) in people with DN versus those without DN. LDL levels in the subgroup analysis stratified by the type of diabetes (B) and symptom severity (C). OR (D) for DN in patients according to serum LDL levels. 
C Study

ID

$\%$

Weight

\section{without pain}

Jeong-Won(2015)

Alma Rosales(2014)

Vincenza(2010)

D. Simmons (1993)

Subtotal (I-squared $=0.0 \%, p=0.733)$

with pain

Jeong-Won(2015)

Jeong-Won(2015)

Alma Rosales(2014)

Vincenza(2010)

D. Simmons (1993)

Subtotal (I-squared $=0.0 \%, p=0.705$ )

Overall (I-squared $=0.0 \%, p=0.582)$

NOTE: Weights are from random effects analysis

WMD (95\% CI)

$\begin{array}{ll}0.02(-0.21,0.25) & 20.98 \\ 0.10(-0.53,0.73) & 279\end{array}$

$-0.08(-0.38,0.22) \quad 12.09$

$0.50(-0.54,1.54) \quad 1.02$

$0.01(-0.16,0.18) \quad 36.88$

$-0.12(-0.31,0.06) \quad 30.80$

$-0.28(-0.48,-0.09) \quad 29.46$

$0.10(-0.76,0.96) \quad 1.50$

$0.08(-1.99,2.14) \quad 0.26$

$0.10(-0.91,1.11) \quad 1.09$

$-0.19(-0.32,-0.06) \quad 63.12$

$-0.12(-0.22,-0.01) \quad 100.00$

D

Study

ID
ES $(95 \% \mathrm{Cl})$

$0.96(0.81,1.14)$

$0.91(0.67,1.21)$

$1.03(0.78,1.37)$

$1.05(0.79,1.40)$

$0.90(0.40,1.90)$

$1.00(0.50,2.10)$

$1.30(0.70,2.60)$

$1.05(0.84,1.30)$

$1.11(0.90,1.37)$

$1.40(1.15,1.71)$

$1.22(1.03,1.45)$

$1.10(1.02,1.19)$

2.6
$\%$

Weight

Figure 5. (continued)

inconsistent results ${ }^{18,28,29,40}$. Here, we performed a systematic review and meta-analysis and found that serum lipid profile changes are correlated with DN. To our knowledge, this is the first meta-analysis to provide evidence of a close relationship between TG, TC, HDL, LDL levels and DN risk.

Exploration of heterogeneity. Because of the apparent heterogeneity of our study, subgroup analysis was performed. We investigated whether the different diabetic types (T1DM or T2DM) and different symptoms (mild and painless or moderate and severe pain) affected the results. These factors may partly explain the origin of heterogeneity.

On the basis of the results, we performed a subgroup analysis with the different diabetic types. In the pooled analysis of the studies, regardless of T1DM or T2DM, the trend of TG change was consistent, and the TG levels of patients with DN were increased compared with those of the control group (Fig. 2B). However, serum HDL levels decreased only in T1DM neuropathy patients, and there was no difference between the control group patients and T2DM neuropathy patients (Fig. 4B). Furthermore, this approach did not obviously change the heterogeneity between the individual efficacy estimates. In addition, another subgroup was performed regarding the different symptoms. Heterogeneity was significantly reduced when analyses were stratified by different symptoms (Fig. 2C, 3C and 5C). We also separately analysed location and study design, as these were assumed to be potential sources of bias (data not shown). These methods slightly change the heterogeneity between the individual efficacy estimates, although they do not eliminate it. 
Due to the limited number of included studies, subgroup analysis could not be conducted to explore the heterogeneity of some outcomes. Other uncharacteristic or unexplained underlying factors may contribute to the heterogeneity.

Relationship between lipid profile and DN. Changes in lipid levels are obvious biological characteristics of DN. After pooled data from 39 studies, our results showed that DN patients had higher levels of serum TG than control patients (Fig. 2A), while HDL levels were lower in DN patients than in control patients (Fig. 4A). The above results indicated that serum detection by TG and HDL may be serological markers for diabetic patients with or without DN. Patients with moderate or severe pain had lower levels of TC and LDL compared to mild or painless DN patients (Figs. 3C and Fig. 5C). It is worth mentioning that TC and LDL level changes in serum may be markers of symptomatic diabetes neuropathy. In addition, the results of our meta-analysis showed that higher levels of TG and LDL significantly increased the risk of DN (Fig. 2D and Fig. 5D). These findings suggest that changes in lipid profiles, especially TG and LDL serum levels, may be risk factors for DN. Highquality randomized controlled trials (RCTs) in the future are needed to better understand the causality between the serum lipid profile and DN.

Underlying mechanisms of lipid effects on DN. There is a high incidence of dyslipidaemia in both T1DM and T2DM patients, and dyslipidaemia is linked to DN (Figs. 2, 3, 4, 5). The mechanisms by which plasma lipids influence DN have not been fully elucidated, but certain factors may be involved. First, patients with dyslipidaemia are characterized by insulin resistance and a chronic inflammation status ${ }^{69}$ that can also contribute to insulin resistance ${ }^{70}$. Furthermore, insulin resistance has shown a positive association with peripheral neuropathy ${ }^{71}$.

Second, oxidative stress is an important risk factor for DN. Neurons express scavenger receptors for oxidized LDLs, such as oxidized LDL receptor $1^{72}$. Elevated LDL has increased susceptibility to oxidation and oxidized LDLs (oxLDLs), and these modified LDLs can bind to extracellular receptors, triggering signalling cascades that activate oxidative stress. oxLDL-induced oxidative stress has been shown to mediate nerve injury in a mouse model of dyslipidaemia-induced neuropathy ${ }^{73}$. In addition, oxLDL is involved in neuron injury through nicotinamide adenine dinucleotide phosphate (NADPH) oxidase activation, which leads to increased superoxide production ${ }^{73}$. Additionally, free fatty acids (FFAs) bind to excess intramitochondrial pyruvate, leading to the production of reactive oxygen species. FFAs have been shown to directly cause damage to Schwann cells in vitro, and they can also cause proinflammatory factors to be released from adipocytes and macrophages ${ }^{71}$.

Third, demyelination due to lipid profile disorders is another potential mechanism for lipid-induced nerve injury. Segmental demyelination is an important feature of DN patients, and myelin breakdown with focal demyelination has been shown to occur in high-fat fed mice ${ }^{74}$. Thus, it is plausible to suggest that dyslipidaemia negatively impacts myelination status in nerves and contributes to the development of DN. Moreover, some studies have indicated that TC can be oxidized to oxysterols, which have been shown to lead to neuronal apoptosis ${ }^{72,75}$.

Of these, insulin resistance, inflammation, oxidative stress, and demyelination are possible mechanisms linking lipid profile disorder to DN.

Surprisingly, TC and LDL serum levels were reduced in patients with severe pain compared with asymptomatic conditions. However, at present, there is no reasonable explanation to explain this phenomenon.

Why intensive blood glucose control has little influence in patients with T2DM. Hyperglycaemia is a key factor underlying DN, but other changes also contribute, including dyslipidaemia and changes in insulin signalling ${ }^{17}$.

Patients with type 2 diabetes have an elevated incidence of dyslipidaemia, which is associated with the occurrence of DN. A study demonstrated that obesity, LDL, HDL, and hypertriglyceridemia were independently associated with neuropathy ${ }^{17}$. A series of bioinformatics analyses identified 532 differentially expressed genes between patient samples with progressive versus nonprogressive diabetic polyneuropathy and found that these were functionally enriched in pathways involving lipid metabolism and inflammatory responses ${ }^{76}$.

T2DM patients are insulin resistant. Disruption of insulin signalling due to insulin resistance makes neurons more vulnerable to metabolic insults and may contribute to the development of $\mathrm{DN}^{77}$.

Hyperglycaemia and dyslipidaemia, together with altered insulin signalling, lead to several pathological alterations in neurons, glia and vascular cells, which can lead to nerve dysfunction and ultimately DN.

Theoretical and practical implications. Our meta-analysis provides good guidance for clinical and scientific research. The treatment of DN has largely been directed at the control of symptoms and glucose control rather than to treat the underlying mechanisms ${ }^{17}$. To date, clinical interventions to treat DN have mainly focused on glycaemic control, while even proper control of blood sugar is a poor efficacy for T2DM neuropathy patients ${ }^{17}$. Along with substantial research into the relationship between serum lipid profiles and DN, the results of our present meta-analytic investigation indicate a clear direction: high levels of TG and LDL increased the risk of DN. In addition, DN patients had higher serum TG and lower HDL levels. Therefore, routine examination of serum TG and HDL levels in diabetic patients to predict the risk of DN is essential. Elevated levels of TG and low levels of HDL may be biomarkers for DN, except for the influence of lifestyle and other factors on blood lipid levels. The clinical use of lipid-lowering drugs may be an effective way to prevent and treat DN. PDN is associated with considerable morbidity, mortality and diminished quality of life ${ }^{9}$. The results of our meta-analysis showed that the presence of decreased TC and LDL in patients with DN may indicate a transition from asymptomatic status to severe pain status in DN patients. To the best of our knowledge, this is the first meta-analysis to explore the relationship between DN and serum lipid profiles; the results may be helpful for future clinical diagnosis 

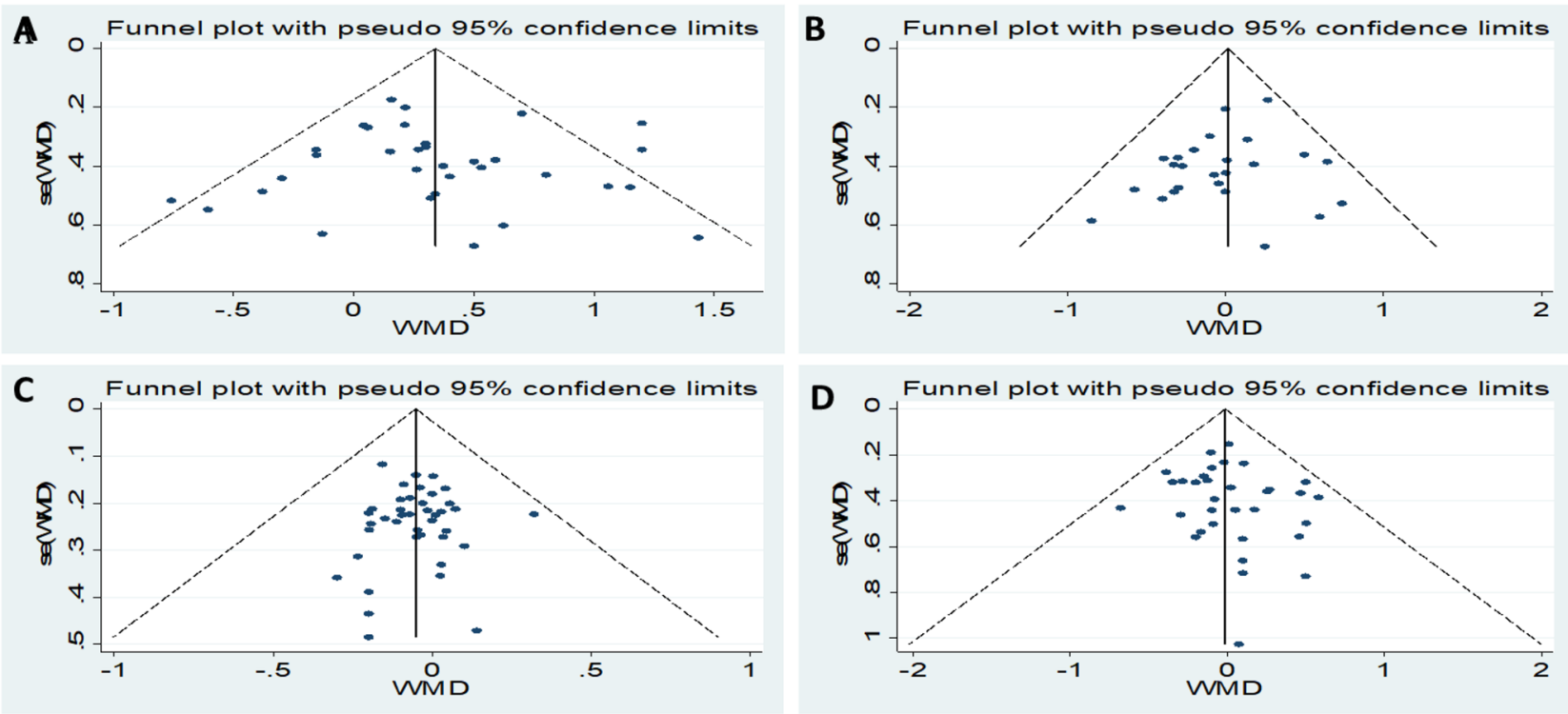

Figure 6. Publication bias funnel plots of the MD for (A) TG, (B) TC, (C) HDL, and (D) LDL and DN.
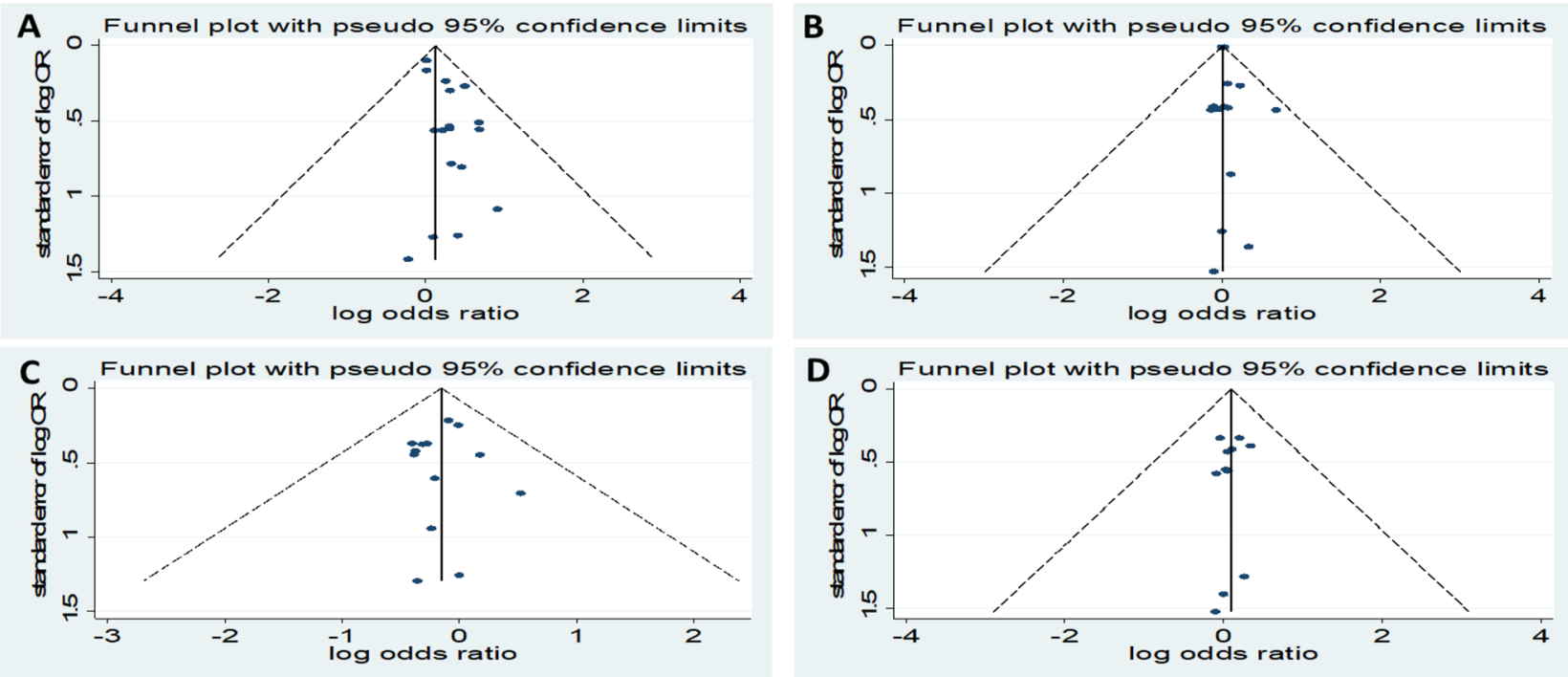

Figure 7. Publication bias funnel plots of the OR for (A) TG, (B) TC, (C) HDL, and (D) LDL and DN.

and treatment. Blood lipid profiles are an appropriate source of biomarkers for DN screening; additionally, these profiles are widely applicable and easily measured.

The implication of our meta-analysis for scientific research is as follows: first, since the included primary studies had different cut-off values of serum lipids, we strongly suggest that the cut-off value be made uniform in subsequent studies. Second, blood lipid levels can be affected by many conditions, such as the environment, lifestyle and diet, and more detailed characteristics of patients should be recorded. Third, TG, TC, HDL and LDL levels provided a state of lipid levels. However, the other parameters may be considered; for instance, TC/HDL and LDL/HDL ratios are also considered indexes for the prediction of DN. Finally, identifying the mechanism of $\mathrm{DN}$ is the basis of treating $\mathrm{DN}$.

Limitations of our study. Our current meta-analysis provides stable evidence of the relationship between the serum lipid profile and DN. Several limitations should be recognized. First, we found significant heterogeneity in the relationship between serum lipid profile and DN risk, which might result from a very large number of included studies and differences in study quality and basic participant characteristics. Second, the mechanisms underlying the decline in serum TC and LDL levels in moderate or severe pain are unclear. Third, there is a shortage of RCTs investigating the impact of lipid levels on DN risk. Thus, in order to obtain a more precise assessment of the impact of lipids on DN risk, well-designed RCTs are necessary. Fourth, DN represents a het- 
A

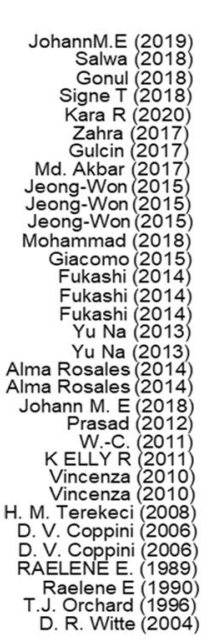

Meta-analysis estimates, given named study is omitted

| Lower Cl Limit OEstimate
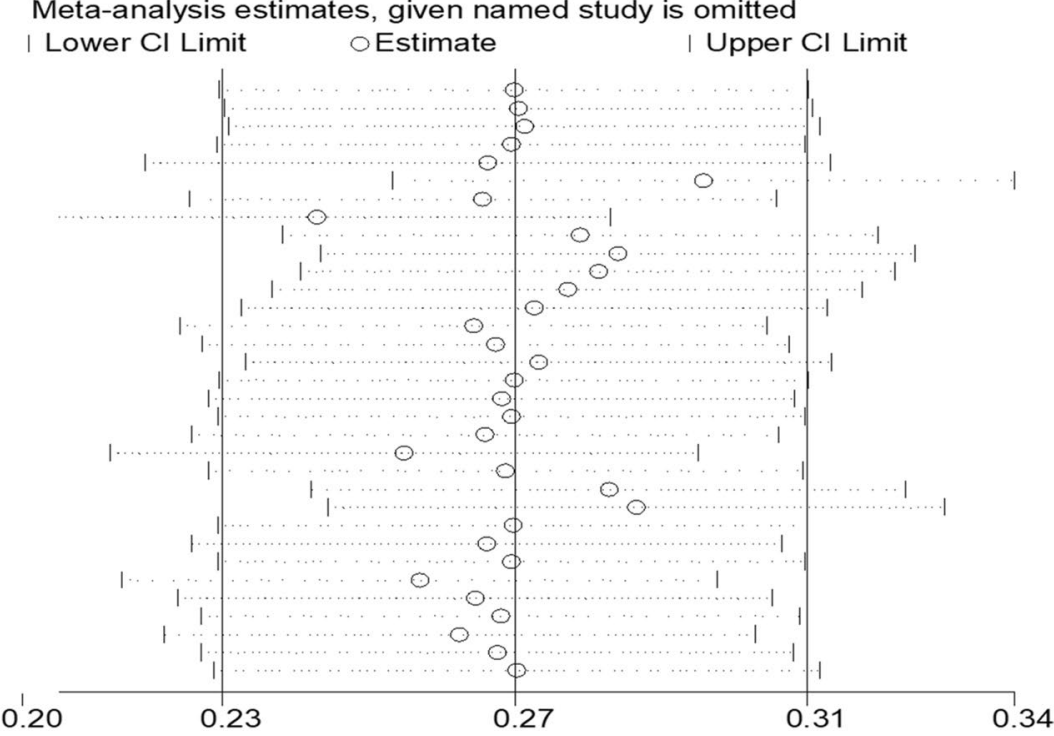

B

Meta-analysis estimates, given named study is omitted

$$
\begin{array}{r}
\text { Kara R (2020) } \\
\text { JohannM.E (2019) } \\
\text { Salwa (2018) } \\
\text { Gonul (2018) } \\
\text { Signe T (2018) } \\
\text { Zahra (2017) } \\
\text { Gulcin (2017) } \\
\text { Md. Akbar (2017) } \\
\text { H. Serife (2016) } \\
\text { Jeong-Won (2015) } \\
\text { Jeong-Won (2015) } \\
\text { Jeong-Won (2015) } \\
\text { Mohammad (2018) } \\
\text { Yu Na (2013) } \\
\text { Yu Na (2013) } \\
\text { Alma Rosales (2014) } \\
\text { Alma Rosales (2014) } \\
\text { Johann M. E (2018) } \\
\text { A. Gordon (2013) } \\
\text { Prasad (2012) } \\
\text { W.-C. (2011) } \\
\text { H. M. Terekeci (2008) } \\
\text { D. V. Coppini (2006) } \\
\text { D. V. Coppini (2006) } \\
\text { D. R. Witte (2004) }
\end{array}
$$

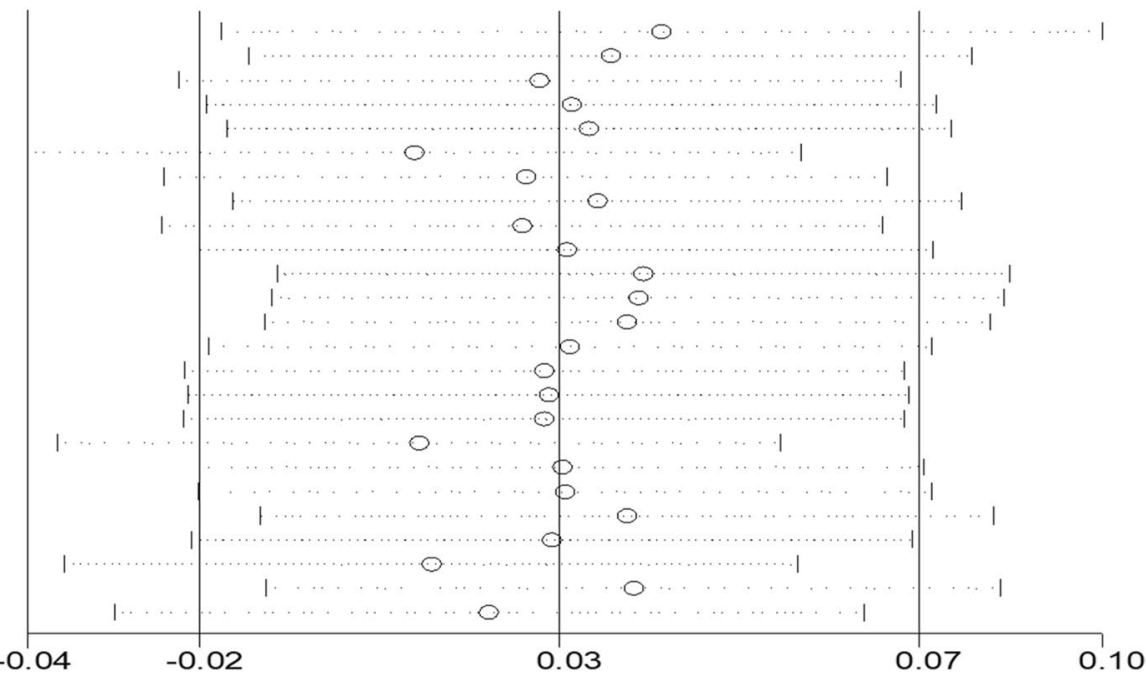

Figure 8. Sensitivity analysis of the pooled MD for (A) TG, (B) TC, (C) HDL, and (D) LDL. 


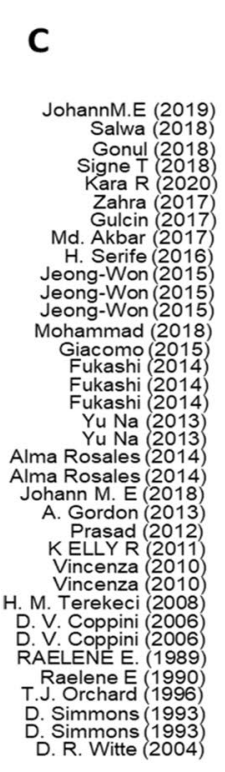

Meta-analysis estimates, given named study is omitted

| Lower $\mathrm{Cl}$ Limit o Estimate

| Upper Cl Limit

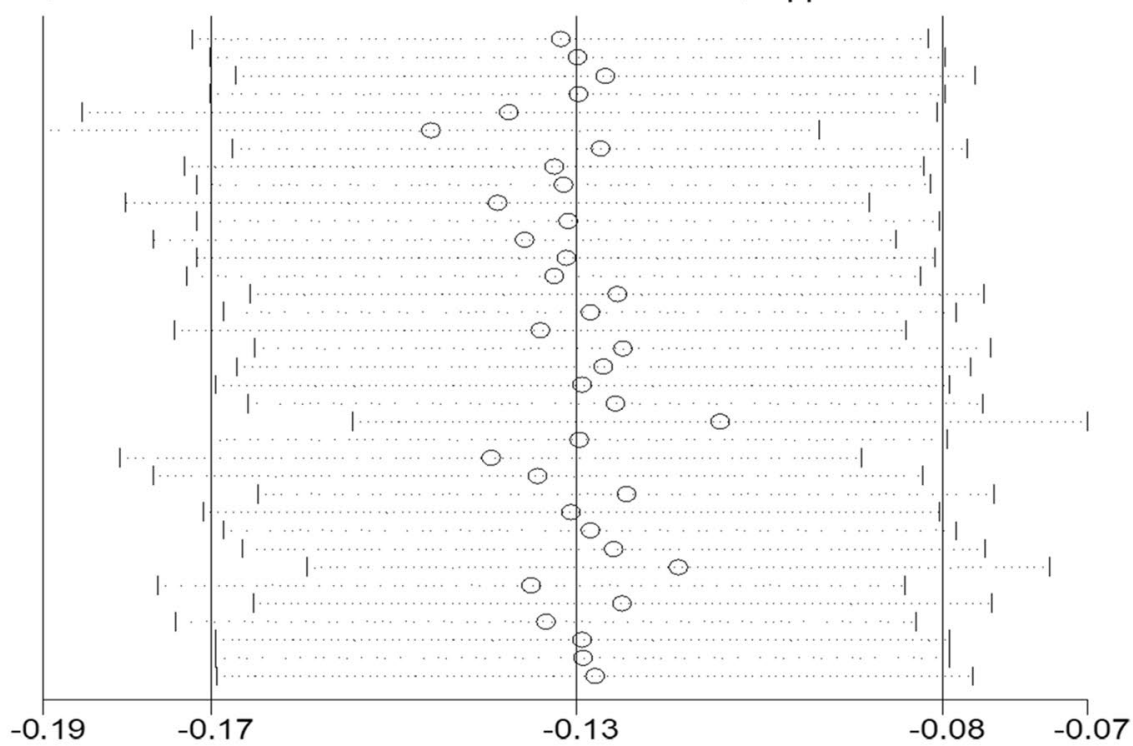

\section{D}

Meta-analysis estimates, given named study is omitted

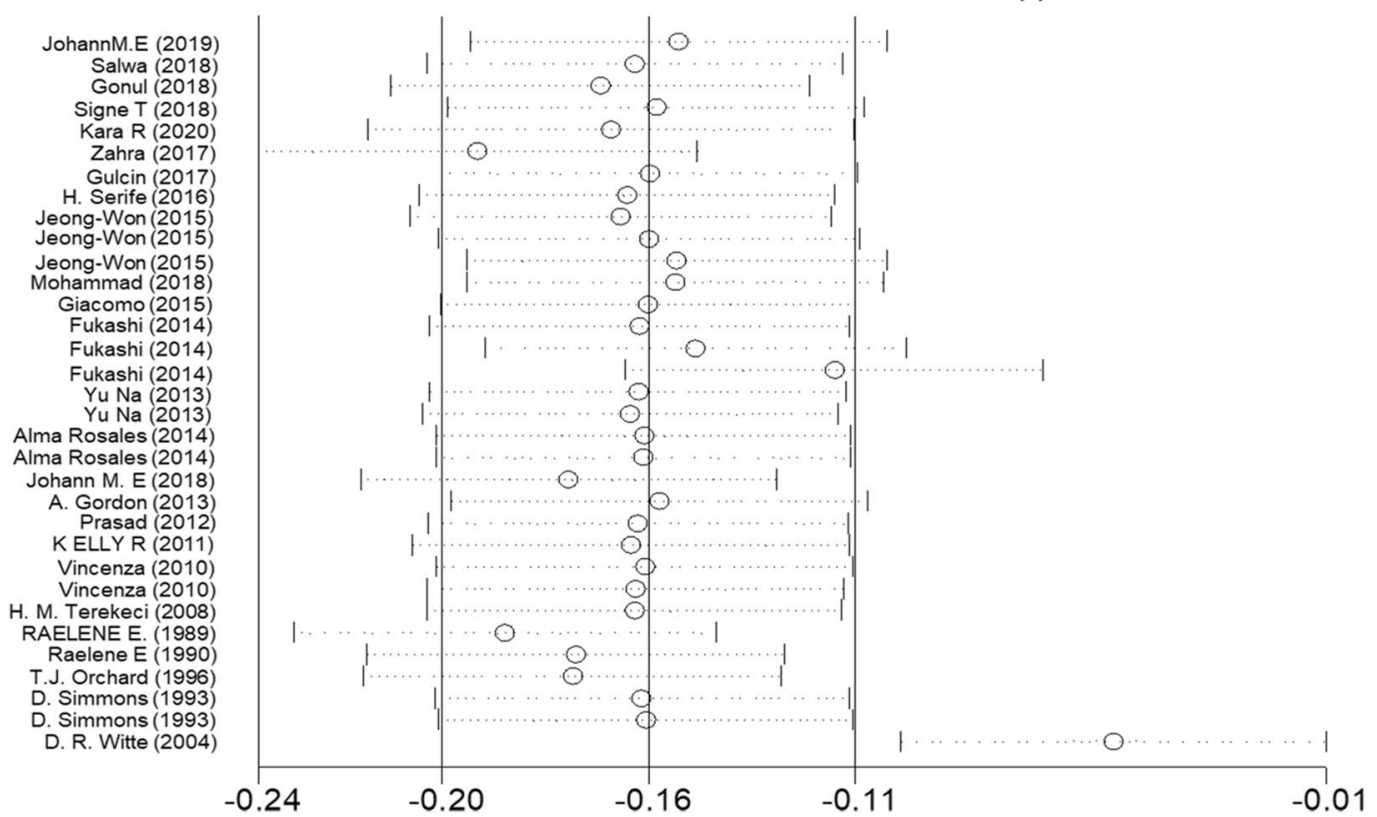

Figure 8. (continued)

erogeneous syndrome, and various definitions and methods are used to formulate the diagnosis. Screening tests for subjective symptoms or objective instrumental assessments, such as vibration perception threshold measurement, are used in the diagnosis of $\mathrm{DN}^{74}$. This increases the heterogeneity between studies. Finally, the included studies were all non-RCTs; thus, causality cannot be shown.

\section{Conclusion}

This meta-analysis supports that higher TG and lower HDL levels may be markers for predicting the development of DN in diabetic patients. Higher TG and LDL levels increase the risk of DN. Reduced TC and LDL serum levels in patients with DN may be a marker of an asymptomatic condition to severe pain condition in patients with DN. The mechanisms underlying the effect of serum TG and LDL levels on the increased risk of DN in recent tobacco quitters need to be further explored, since an improved understanding of this effect could contribute to the development of targeted pharmaceuticals that could be used to aid lipid profile regulation and prediction or treatment of DN. 
A

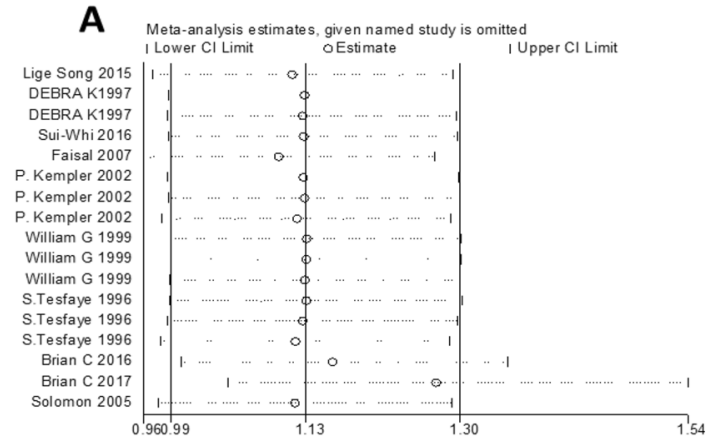

C Meta-analysis estimates, given named study is omitted

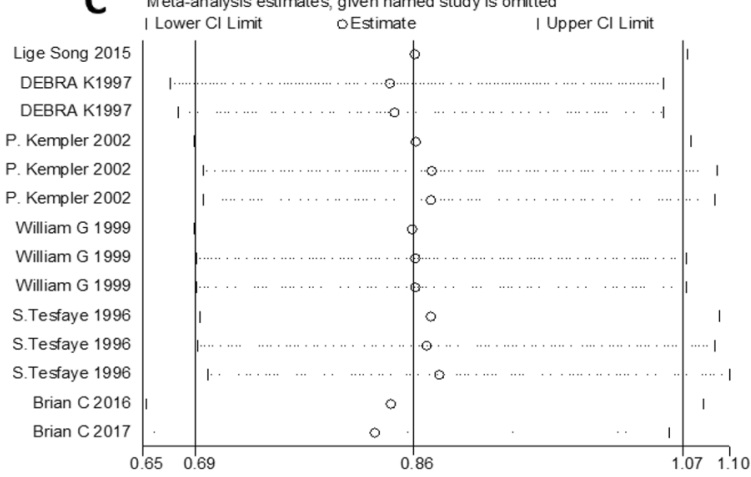

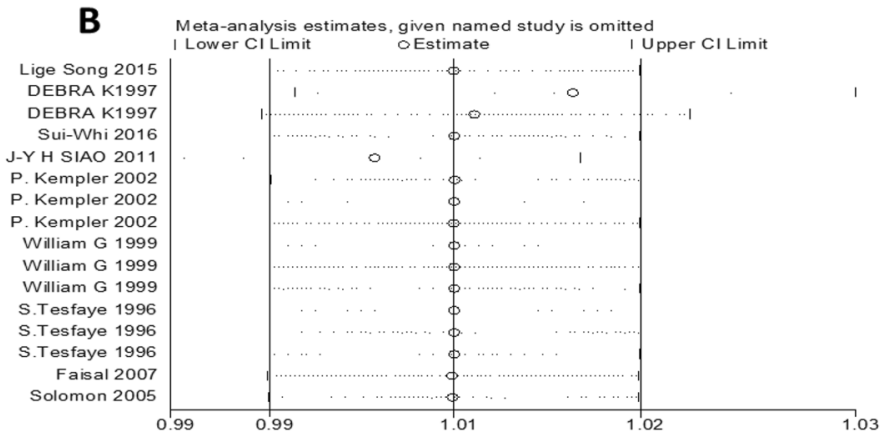

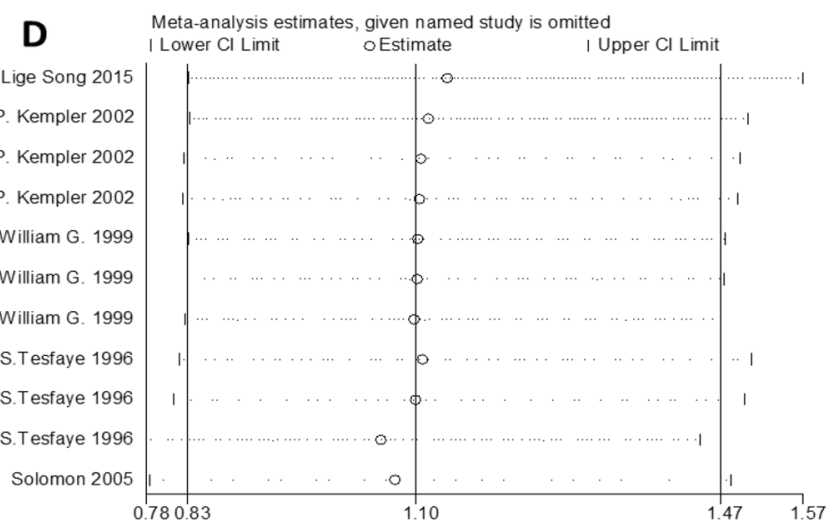

Figure 9. Sensitivity analysis of the pooled OR for (A) TG, (B) TC, (C) HDL, and (D) LDL.

Received: 10 June 2020; Accepted: 4 December 2020

Published online: 12 January 2021

\section{References}

1. Callaghan, B. C., Price, R. S. \& Feldman, E. L. Distal symmetric polyneuropathy: a review. JAMA 314(20), 2172-2181 (2015).

2. Savettieri, G. et al. Prevalence of diabetic neuropathy with somatic symptoms: a door-to-door survey in two Sicilian municipalities. Sicilian Neuro-Epidemiologic Study (SNES) Group. Neurology 43(6), 1115-1120 (1993).

3. Bharucha, N. E., Bharucha, A. E. \& Bharucha, E. P. Prevalence of peripheral neuropathy in the Parsi community of Bombay. Neurology 41(8), 1315-1317 (1991).

4. Gregg, E. W. et al. Prevalence of lower-extremity disease in the US adult population $>=40$ years of age with and without diabetes: 1999-2000 national health and nutrition examination survey. Diabetes Care 27(7), 1591-1597 (2004).

5. Soedamah-Muthu, S. S. et al. Relationship between risk factors and mortality in type 1 diabetic patients in Europe: the EURODIAB Prospective Complications Study (PCS). Diabetes Care 31(7), 1360-1366 (2008).

6. Nather, A. et al. Epidemiology of diabetic foot problems and predictive factors for limb loss. J. Diabetes Complic. 22(2), 77-82 (2008).

7. Kioskli, K. et al. Psychosocial factors in painful diabetic neuropathy: a systematic review of treatment trials and survey studies. Pain Med. 20(9), 1756-1773 (2019).

8. Costa, A. F. et al. Burden of type 2 diabetes mellitus in Brazil. Cad SaudePublica 33(2), e00197915 (2017).

9. Tesfaye, S. Recent advances in the management of diabetic distal symmetrical polyneuropathy. J. Diabetes Investig. 2(1), 33-42 (2011).

10. Davies, M. et al. The prevalence, severity, and impact of painful diabetic peripheral neuropathy in type 2 diabetes. Diabetes Care 29(7), 1518-1522 (2006)

11. Gore, M. et al. Pain severity in diabetic peripheral neuropathy is associated with patient functioning, symptom levels of anxiety and depression, and sleep. J. Pain Symptom Manag. 30(4), 374-385 (2005).

12. Vinik, A. et al. Relationship between pain relief and improvements in patient function/quality of life in patients with painful diabetic peripheral neuropathy or postherpetic neuralgia treated with pregabalin. Clin. Ther. 35(5), 612-623 (2013).

13. Jose, V. M. et al. Randomized double-blind study comparing the efficacy and safety of lamotrigine and amitriptyline in painful diabetic neuropathy. Diabet Med. 24(4), 377-383 (2007).

14. Morello, C. M. et al. Randomized double-blind study comparing the efficacy of gabapentin with amitriptyline on diabetic peripheral neuropathy pain. Arch. Intern. Med. 159(16), 1931-1937 (1999).

15. Ohkubo, Y. et al. Intensive insulin therapy prevents the progression of diabetic microvascular complications in Japanese patients with non-insulin-dependent diabetes mellitus: a randomized prospective 6-year study. Diabetes Res. Clin. Pract. 28(2), 103-117 (1995).

16. Linn, T. et al. Intensive therapy in adult insulin-dependent diabetes mellitus is associated with improved insulin sensitivity and reserve: a randomized, controlled, prospective study over 5 years in newly diagnosed patients. Metabolism 45(12), 1508-1513 (1996).

17. Callaghan, B. C. et al. Diabetic neuropathy: clinical manifestations and current treatments. Lancet Neurol. 11(6), 521-534 (2012).

18. Tesfaye, S. et al. Prevalence of diabetic peripheral neuropathy and its relation to glycaemic control and potential risk factors: the EURODIAB IDDM Complications Study. Diabetologia 39(11), 1377-1384 (1996).

19. Ziegler, D. et al. Prevalence of polyneuropathy in pre-diabetes and diabetes is associated with abdominal obesity and macroangiopathy: the MONICA/KORA Augsburg Surveys S2 and S3. Diabetes Care 31(3), 464-469 (2008). 
20. Wiggin, T. D. et al. Elevated triglycerides correlate with progression of diabetic neuropathy. Diabetes 58(7), 1634-1640 (2009).

21. Khovidhunkit, W. et al. Effects of infection and inflammation on lipid and lipoprotein metabolism: mechanisms and consequences to the host. J. Lipid Res. 45(7), 1169-1196 (2004).

22. Smith, A. G. \& Singleton, J. R. Obesity and hyperlipidemia are risk factors for early diabetic neuropathy. J. Diabetes Complic. 27(5), 436-442 (2013).

23. Hwang, J. W., Pyun, S. B. \& Kwon, H. K. Relationship of vascular factors on electrophysiologic severity of diabetic neuropathy. Ann. Rehabil. Med. 40(1), 56-65 (2016).

24. Hsu, W. C. et al. Somatic neuropathy is an independent predictor of all- and diabetes-related mortality in type 2 diabetic patients: a population-based 5-year follow-up study (KCIS No. 29). Eur. J. Neurol. 19(9), 1192-1198 (2012).

25. Akbar, M. et al. Potential association of triglyceride glucose index with cardiac autonomic neuropathy in type 2 diabetes mellitus patients. J. Korean Med. Sci. 32(7), 1131-1138 (2017).

26. Aktaş, H. S et al. The relation of protein $\mathrm{C}$ and protein $\mathrm{S}$ levels with cardiovascular risk in patients with diabetic neuropathy. Diabetes Metab. Syndr. 10(4), 234-237 (2016).

27. Al-Mahroos, F. \& Al-Roomi, K. Diabetic neuropathy, foot ulceration, peripheral vascular disease and potential risk factors among patients with diabetes in Bahrain: a nationwide primary care diabetes clinic-based study. Ann. Saudi Med. 27(1), 25-31 (2007).

28. Kempler, P. et al. Autonomic neuropathy is associated with increased cardiovascular risk factors: the EURODIAB IDDM Complications Study. Diabet. Med. 19(11), 900-909 (2002).

29. Song, L., Zhou, L. \& Tang, Z. An association analysis of lipid profile and diabetic cardiovascular autonomic neuropathy in a Chinese sample. Lipids Health Dis. 15, 122 (2016).

30. Swartz, M. K. The PRISMA statement: a guideline for systematic reviews and meta-analyses. J. Pediatr. Health Care 25(1), 1-2 (2011).

31. Stang, A. Critical evaluation of the Newcastle-Ottawa scale for the assessment of the quality of nonrandomized studies in metaanalyses. Eur. J. Epidemiol. 25(9), 603-605 (2010).

32. Sabbagh, H. J. et al. Passive smoking in the etiology of non-syndromicorofacial clefts: a systematic review and meta-analysis. PLoS ONE 10(3), e0116963 (2015).

33. Hozo, S. P., Djulbegovic, B. \& Hozo, I. Estimating the mean and variance from the median, range, and the size of a sample. $B M C$ Med. Res. Methodol. 5, 13 (2005).

34. Patsopoulos, N. A., Evangelou, E. \& Ioannidis, J. P. Sensitivity of between-study heterogeneity in meta-analysis: proposed metrics and empirical evaluation. Int. J. Epidemiol. 37(5), 1148-1157 (2008).

35. Jende, J. et al. Association of serum cholesterol levels with peripheral nerve damage in patients with type 2 diabetes. JAMA Netw. Open 2(5), e194798 (2019).

36. Hosny, S. S. et al. Relation between plasma Apelin level and peripheral neuropathy in Type 2 diabetic patients. Diabetes Metab. Syndr. 13(1), 626-629 (2019).

37. Vural, G. \& Gümüsyayla, Ş. Monocyte-to-high density lipoprotein ratio is associated with a decreased compound muscle action potential amplitude in patients with diabetic axonal polyneuropathy. Medicine (Baltimore) 97(42), e12857 (2018).

38. Andersen, S. T. et al. Corneal confocal microscopy as a tool for detecting diabetic polyneuropathy in a cohort with screen-detected type 2 diabetes: ADDITION-Denmark. J. Diabetes Complic. 32(12), 1153-1159 (2018).

39. Mizokami-Stout, K. R. et al. The contemporary prevalence of diabetic neuropathy in type 1 diabetes: findings from the T1D exchange. Diabetes Care 43(4), 806-812 (2020).

40. Litzelman, D. K., Marriott, D. J. \& Vinicor, F. Independent physiological predictors of foot lesions in patients with NIDDM. Diabetes Care 20(8), 1273-1278 (1997).

41. Aryan, Z. et al. Conflicting interactions of apolipoprotein A and high density lipoprotein cholesterol with microvascular complications of type 2 diabetes. Diabetes Res. Clin. Pract. 133, 131-141 (2017).

42. Akinci, G. et al. Clinical spectra of neuromuscular manifestations in patients with lipodystrophy: a multicenter study. Neuromuscul. Disord. 27(10), 923-930 (2017).

43. Jane, S. W. et al. Prevalence, discomfort and self-relief behaviours of painful diabetic neuropathy in Taiwan: a cross-sectional study. BMJ Open 6(10), e011897 (2016)

44. Najafi, M. T. et al. Ambulatory blood pressure monitoring and diabetes complications: targeting morning blood pressure surge and nocturnal dipping. Medicine (Baltimore) 97(38), e12185 (2018).

45. Zoppini, G. et al. Prevalence of cardiovascular autonomic neuropathy in a cohort of patients with newly diagnosed type 2 diabetes: the Verona newly diagnosed type 2 diabetes study (VNDS). Diabetes Care 38(8), 1487-1493 (2015).

46. Ishibashi, F. et al. Correlation between sudomotor function, sweat gland duct size and corneal nerve fiber pathology in patients with type 2 diabetes mellitus. J. Diabetes Investig. 5(5), 588-596 (2014).

47. Cho, Y. N. et al. The role of insulin resistance in diabetic neuropathy in Koreans with type 2 diabetes mellitus: a 6-year follow-up study. Yonsei Med. J. 55(3), 700-708 (2014).

48. Rosales-Hernandez, A. et al. Absence of clinical relationship between oxidized low density lipoproteins and diabetic peripheral neuropathy: a case control study. Lipids Health Dis. 13, 32 (2014).

49. Jende, J. et al. Diabetic neuropathy differs between type 1 and type 2 diabetes: insights from magnetic resonance neurography. Ann. Neurol. 83(3), 588-598 (2018).

50. Katulanda, P. et al. The prevalence, patterns and predictors of diabetic peripheral neuropathy in a developing country. Diabetol. Metab. Syndr. 4(1), 21 (2012).

51. Hsiao, J. Y. et al. The relationship between diabetic autonomic neuropathy and diabetic risk factors in a Taiwanese population. J. Int. Med. Res. 39(4), 1155-1162 (2011).

52. Ylitalo, K. R., Sowers, M. \& Heeringa, S. Peripheral vascular disease and peripheral neuropathy in individuals with cardiometabolic clustering and obesity: National Health and Nutrition Examination Survey 2001-2004. Diabetes Care 34(7), 1642-1647 (2011).

53. Spallone, V. et al. Clinical correlates of painful diabetic neuropathy and relationship of neuropathic pain with sensorimotor and autonomic nerve function. Eur. J. Pain 15(2), 153-160 (2011).

54. Nybo, M. et al. Plasma osteoprotegerin concentrations in peripheral sensory neuropathy in Type 1 and Type 2 diabetic patients. Diabet. Med. 27(3), 289-294 (2010).

55. Coppini, D. V. et al. Established diabetic neuropathy seems irreversible despite improvements in metabolic and vascular risk markers-a retrospective case-control study in a hospital patient cohort. Diabet. Med. 23(9), 1016-1020 (2006).

56. Witte, D. R. et al. Risk factors for cardiac autonomic neuropathy in type 1 diabetes mellitus. Diabetologia 48(1), 164-171 (2005).

57. Maser, R. E. et al. Epidemiological correlates of diabetic neuropathy. Report from Pittsburgh Epidemiology of Diabetes Complications Study. Diabetes 38(11), 1456-1461 (1989).

58. Maser, R. E. et al. Diabetic autonomic neuropathy and cardiovascular risk. Pittsburgh Epidemiology of Diabetes Complications Study III. Arch. Intern. Med. 150(6), 1218-1222 (1990).

59. Orchard, T. J. et al. Why does diabetic autonomic neuropathy predict IDDM mortality? An analysis from the Pittsburgh Epidemiology of Diabetes Complications Study. Diabetes Res. Clin. Pract. 34(Suppl), S165-S171 (1996).

60. Simmons, D., Ng, L. L. \& Bomford, J. Relationship between myoinositol influx and lipids in diabetic neuropathy. ActaDiabetol. 30(4), 233-237 (1993). 
61. Sone, H., S. Mizuno and N. Yamada, Vascular risk factors and diabetic neuropathy. N Engl J Med, 2005. 352(18): p. 1925-7; author reply $1925-7$.

62. Callaghan, B. C. et al. Metabolic syndrome components are associated with symptomatic polyneuropathy independent of glycemic status. Diabetes Care 39(5), 801-807 (2016).

63. Callaghan, B. C. et al. Diabetes and obesity are the main metabolic drivers of peripheral neuropathy. Ann. Clin. Transl. Neurol. 5(4), 397-405 (2018)

64. Christen, W. G. et al. Risk factors for progression of distal symmetric polyneuropathy in type 1 diabetes mellitus. Sorbinil Retinopathy Trial Research Group. Am. J. Epidemiol. 150(11), 1142-1151 (1999).

65. Terekeci, H. M. et al. Plasma osteoprotegerin concentrations in type 2 diabetic patients and its association with neuropathy. Exp. Clin. Endocrinol. Diabetes 117(3), 119-123. https://doi.org/10.1055/s-0028-1085425 (2009).

66. Vinik, A. I. et al. Diabetic neuropathies. Diabetologia 43(8), 957-973 (2000).

67. Pop-Busui, R. et al. Diabetic neuropathy: a position statement by the American Diabetes Association. Diabetes Care 40(1), 136-154 (2017).

68. The effect of intensive diabetes therapy on the development and progression of neuropathy. The Diabetes Control and Complications Trial Research Group. Ann Intern Med, 1995. 122(8): 561-568.

69. Sears, B. \& Perry, M. The role of fatty acids in insulin resistance. Lipids Health Dis. 14, 121 (2015).

70. Kim, J.J. and D.D. Sears, TLR4 and Insulin Resistance. Gastroenterol Res Pract, 2010. 2010.

71. Han, L. et al. Peripheral neuropathy is associated with insulin resistance independent of metabolic syndrome. Diabetol. Metab. Syndr. 7, 14 (2015).

72. Vincent, A. M. et al. Diabetic neuropathy: cellular mechanisms as therapeutic targets. Nat. Rev. Neurol. 7(10), 573-583 (2011).

73. Vincent, A. M. et al. Dyslipidemia-induced neuropathy in mice: the role of oxLDL/LOX-1. Diabetes 58(10), 2376-2385 (2009).

74. Xie, F. et al. High energy diets-induced metabolic and prediabetic painful polyneuropathy in rats. PLoS ONE 8(2), e57427 (2013).

75. Jang, E. R. \& Lee, C. S. 7-ketocholesterol induces apoptosis in differentiated PC12 cells via reactive oxygen species-dependent activation of NF- $\kappa \mathrm{B}$ and Akt pathways. Neurochem. Int. 58(1), 52-59 (2011).

76. Hur, J. et al. The identification of gene expression profiles associated with progression of human diabetic neuropathy. Brain 134(Pt 11), 3222-3235 (2011).

77. Kim, B. et al. Hyperinsulinemia induces insulin resistance in dorsal root ganglion neurons. Endocrinology 152(10), 3638-3647 (2011).

\section{Author's contributions}

J.Z. coordinated the study. Z.C. developed the idea for the study and, along with Y.Y. and J.Z., contributed to the study design, literature search, figures, statistical analysis, outcome data synthesis, and drafting and editing of the final paper. All authors critically revised the report. All members have approved and agreed to submit the manuscript.

\section{Funding}

This work was supported by grants from the National Natural Science Foundation of China $(82070807,91749118$, 81770775, 81730022), the Planned Science and Technology Project of Hunan Province (2017RS3015) and National key research and development program (2019YFA0801903, 2018YFC2000100).

\section{Competing interests}

The authors declare no competing interests.

\section{Additional information}

Supplementary Information is available for this paper at https://doi.org/10.1038/s41598-020-79276-0.

Correspondence and requests for materials should be addressed to J.Z.

Reprints and permissions information is available at www.nature.com/reprints.

Publisher's note Springer Nature remains neutral with regard to jurisdictional claims in published maps and institutional affiliations.

(c) (1) Open Access This article is licensed under a Creative Commons Attribution 4.0 International (c) License, which permits use, sharing, adaptation, distribution and reproduction in any medium or format, as long as you give appropriate credit to the original author(s) and the source, provide a link to the Creative Commons licence, and indicate if changes were made. The images or other third party material in this article are included in the article's Creative Commons licence, unless indicated otherwise in a credit line to the material. If material is not included in the article's Creative Commons licence and your intended use is not permitted by statutory regulation or exceeds the permitted use, you will need to obtain permission directly from the copyright holder. To view a copy of this licence, visit http://creativecommons.org/licenses/by/4.0/.

(C) The Author(s) 2021 